\title{
A particle-in-cell method for the simulation of plasmas based on an unconditionally stable field solver
}

\author{
Eric M. Wolf ${ }^{\mathrm{a}, *}$, Matthew Causley ${ }^{\mathrm{b}}$, Andrew Christlieb ${ }^{\mathrm{a}}$, Matthew Bettencourt ${ }^{\mathrm{c}}$ \\ ${ }^{a}$ Department of Mathematics, Michigan State University, East Lansing, MI 48824 \\ ${ }^{b}$ Department of Mathematics, Kettering University, Flint, MI 48504 \\ ${ }^{c}$ Sandia National Laboratory, Albuquerque, NM 87185
}

\begin{abstract}
We propose a new particle-in-cell (PIC) method for the simulation of plasmas based on a recently developed, unconditionally stable solver for the wave equation. This method is not subject to a CFL restriction, limiting the ratio of the time step size to the spatial step size, typical of explicit methods, while maintaining computational cost and code complexity comparable to such explicit schemes. We describe the implementation in one and two dimensions for both electrostatic and electromagnetic cases, and present the results of several standard test problems, showing good agreement with theory with time step sizes much larger than allowed by typical CFL restrictions.
\end{abstract}

Keywords:

computational plasma physics, unconditionally stable field solver, particle-in-cell

\section{Introduction}

Collisionless plasmas - systems of charged particles interacting through electromagnetic fields - are modelled by the Vlasov-Maxwell system of partial differential equations (PDEs), which couple Maxwell's equations, describing the evolution of the electric and magnetic fields $\mathbf{E}$ and $\mathbf{B}$, to Vlasov equations, a type of hyperbolic PDE describing the evolution of the phase-space distribution functions (DFs) $f_{s}$ of the various species $s$ of charged particles. Particle-in-cell (PIC) methods [1, 2, 3], in development and use since the 1960s and a primary tool in the computer simulation of plasmas, combine an Eulerian description of the fields with a Lagrangian description of the DFs; that is, fields are evolved on a fixed grid, while DFs are

\footnotetext{
${ }^{*}$ Corresponding author

Email addresses: wolferi1@msu.edu (Eric M. Wolf), mcausley@kettering.edu (Matthew Causley), andrewch@math.msu.edu (Andrew Christlieb), mbetten@sandia.gov (Matthew Bettencourt)

${ }^{1}$ Sandia is a multiprogram laboratory operated by Sandia Corporation, a Lockheed Martin Company, for the United States Department of Energy's National Nuclear Security Administration under contract DE-AC04-94AL85000.

${ }^{2}$ This work has been supported in part by AFOSR grants FA9550-11-1-0281, FA9550-12-1-0343 and FA9550-12-1-0455, NSF grant DMS-1115709, and MSU Foundation grant SPG-RG100059.
} 
represented by moving particles whose trajectories are characteristics of the corresponding Vlasov equation. Thus, PIC methods require a method to compute the fields on a grid and a method to compute particle trajectories, as well as interpolation tools to provide for their coupling. This work focuses on a new method for the computation of the fields, along with associated interpolation techniques.

Under the Lorenz gauge condition, Maxwell's equations reduce to uncoupled wave equations for the scalar and vector potentials, $\Phi$ and $\mathbf{A}$. Recently, a novel method for the solution of the wave equation has been developed [4, 5, 6], based on the Method of Lines Transpose (MOLT), dimensional splitting and an efficient 1D integral solution method, which is unconditionally stable (or A-stable) - that is, it is not subject to the Courant-Friedrichs-Lewy (CFL) restriction limiting the ratio of the temporal step size to the spatial step size, typical of widely used explicit methods. In this work, we apply this method to the uncoupled wave equations for $\Phi$ and A to solve Maxwell's equations with a method comparable in computational cost and complexity of code to explicit methods such as the well-known finite difference time-domain (FDTD) method (also known as the Yee scheme) [7, 8], but without introducing a CFL restriction based on the speed of light as in such explicit methods. In conjunction with an appropriate description of particles, we seek to develop a PIC method that retains the simplicity of explicit finite-difference-based methods while eliminating this CFL restriction.

We demonstrate the application of our method to both electrostatic and electromagnetic problems. In the non-relativistic, zero-magnetic field limit, it is typical to make the electrostatic approximation, $\mathbf{E}=-\nabla \Phi,-\nabla^{2} \Phi=\rho / \epsilon_{0}$, simplifying the Vlasov-Maxwell system to the Vlasov-Poisson system. Correspondingly, in this work we consider the same nonrelativistic, zero-magnetic field limit, and drop $\mathbf{A}$ and the corresponding wave equations from our model. When particle velocities are small compared to the speed of light, we argue that this model, which we term the Vlasov-Wave model due to the replacement of the Poisson equation with a wave equation, will agree approximately with the usual electrostatic Vlasov-Poisson model. We present numerical results applying our method to several standard electrostatic test problems in one and two dimensions, showing agreement with the predictions of linear theory for the electrostatic model. For the present work, we consider these electrostatic problems merely as test cases leading to electromagnetic problems, as the numerical solution of the Poisson equation does not typically introduce a CFL restriction, so that one of the chief virtues of our method (the lack of a CFL restriction) is made irrelevant for the simple electrostatic problems considered here. However, in conjunction with embedded boundary and domain decomposition techniques, it may be possible using our method to produce a fast parallel solver for large electrostatic problems with complex geometry that avoids the difficulties of a solving a global linear system, though this is beyond the scope of the present work. We apply our method to a pair of electromagnetic beam pinch problems in two dimensions. It is well known that an electromagnetic PIC (EM-PIC) method must satisfy a discrete form of Gauss' law $\left(\nabla \cdot \mathbf{E}=\rho / \epsilon_{0}\right)$ to prevent serious numerical errors related to the violation of charge conservation [9]. In this work, we obtain solutions that satisfy exactly discrete forms of Gauss' law for the electric field and the divergence-free condition 
for the magnetic field through a staggered grid approach, adapted from the well-known Yee grid [7], with a Poisson equation formulation for the scalar potential. In addition to eliminating the CFL restriction, the wave solver method used in this work offers the handling of complex boundary geometry in a Cartesian grid, demonstrated for the scalar wave equation to second-order accuracy in [6], without using the staircasing approximation of traditional FDTD methods [10], and can be extended to higher-order accuracy [5], features that will be incorporated into our PIC method in future work.

We now review some recent works on numerical methods for EM-PIC simulations, with attention to the areas of complex boundary geometry modeling, grid refinement schemes, and unconditionally stable field solvers. Future work will compare our PIC method to these other newly developed PIC methods. We first direct the reader to the comprehensive review of conformal (body-fitted) EM-PIC algorithms presented in [11], which summarizes many methods for modeling complex boundary geometries beyond the staircasing approximation used in traditional FDTD methods. We now briefly describe some recent works on EM-PIC grid refinement, first recalling the basic issue that short wavelengths supported on the fine but not the coarse grid level may reflect off of coarse-fine grid boundaries and contaminate the fine grid solution.

An EM-PIC grid refinement scheme for an FDTD field solver was presented in [12], which used perfectly matched layers (PMLs) to absorb outgoing waves from grid patches in order to prevent the reflection and buildup of short wavelengths. Fields are evolved on three grids: the initial coarse grid, a fine grid patch, and an auxiliary coarse grid patch coinciding with the fine grid patch. Assuming linear particle shapes, currents are deposited on the fine grid, then propagated to the coarse grids, while fields are evaluated on all grid levels, then summed appropriately, with the fields on the auxiliary coarse grid patch being subtracted to avoid double counting. However, a spurious force related to particles crossing patch boundaries is detected, which the authors indicate is related to the issue of current weighting and charge conservation across grid boundaries. Further, the present authors understand that particles crossing the PML may generate spurious Cerenkov radiation (due to the numerical dispersion relation changing with the PML material properties), so that an alternative high-order absorbing boundary condition may be preferable in some applications.

Another EM-PIC grid refinement scheme for an FDTD field solver was presented in [13], which uses an explicit spatial averaging operation to damp short wavelengths. This scheme is based on quad-/oct-tree Adaptive Mesh Refinement (AMR). Buffer cells surrounding the refined cells are used to supply boundary values, while coarse grid data is overwritten by fine grid data after each time step. A global time step size is used, determined by the CFL restriction based on the finest grid level. Discrete divergence relations are satisfied through use of the Villasenor-Buneman current weighting scheme [14] and specially designed intergrid interpolation operators.

A novel EM-PIC grid refinement scheme was presented in [15], with a 2D implementation presented in [16]. This scheme is distinguished by its use of the implicit moment method [17] and by its multi-level, multi-domain approach to grid refinement, which the authors claim offers greater flexibility in the choice of model and numerical method on each different 
grid level or patch. In the implicit moment method, particle-field coupling is approximated with a moment-particle coupling that allows for the numerical solution, via the Generalized Minimal Residual (GMRES) method, of an implicit second-order equation for the electric field at time level $n+\theta$ with magnetic field and moment terms appearing only as source terms at time level $n$. Gauss' law is enforced by an elliptic divergence cleaning step, and following the computation of the updated electric field, the magnetic field is updated using a discretization of the Maxwell-Faraday equation. The implicit moment method relaxes the time step restrictions of explicit PIC methods and introduces a damping of small wavelengths. Particular attention is paid to the issues surrounding particle weighting on nonuniform grides, which is approached by evolving separate, full PIC simulations on each grid level, with new particles spawning on the fine grid levels as particles enter the corresponding area on the coarse grid. Boundary conditions are interpolated from the updated fields on the coarse grid to the fine grid patches, which the authors identify as a possible bottleneck of future parallel implementations, as field computation on the fine grid patches may need to wait for the coarse grid fields to be updated before proceeding.

A PIC method based on a high-order nodal discontinuous Galerkin scheme for Maxwell's equations, allowing for the handling of complex geometries and grid refinement through use of unstructured grides but still subject to a CFL restriction based on the speed of light, was presented in [18]. It was extended in [19] to include a hyperbolic divergence cleaning scheme with implicit-explicit time-stepping to avoid further restriction of the time step size due to the large wave speed associated with hyperbolic divergence cleaning.

We now review some recent works that present unconditionally stable field solvers. Building on prior work on unconditionally stable ADI-FDTD schemes for Maxwell's equations [20, 21, 22, 23], unconditionally stable ADI-FDTD methods for Maxwell's equations that conserve the divergence of the discrete electric and magnetic fields were incorporated into PIC methods in [24], using the method of [25] to handle complex geometries.

The Pseudo-Spectral Analytical Time-Domain (PSATD) method proposed in [26] uses an FFT-based algorithm with analytical time integration of the field equations in frequency space (over one time step, assuming that, over each time step, current sources are constant and charge density varies linearly in time). Their method is unconditionally stable and offers a high level of control over the numerical dispersion relation via modification of numerical parameters [27], which in [28] and [29] is used in conjunction with a filter to suppress numerical Cerenkov instabilities in relativistic beam simulations. The PSATD method decomposes the domain into rectangular blocks buffered by a layer of guard cells of width $O(c \Delta t)$ to facilitate the application of the FFT, which seems to complicate the implementation of boundary conditions on complex geometries, and to the present authors' knowledge, no such implementation has been presented yet in the literature. The authors suggest various means to achieve the satisfaction of discrete forms of divergence relations: by inclusion of a term involving the time derivative of the charge density (which requires taking the FFT of the charge density at each time step), with an elliptic divergence correction, or by use of the Villasenor-Buneman [14] or Esirkepov [30] charge conserving current weighting schemes. However, their domain decomposition algorithm introduces further sources of 
divergence error in both Gauss law and the divergence free condition on the magnetic field, and an additional hyperbolic divergence cleaning step is applied to both curl equations.

In contrast to the PSATD method, our method, based on physical-space versus frequencyspace computation, does not offer as much direct control over the numerical dispersion relation. However, we can still achieve improved wave propagation properties with our method by using higher-order accurate versions of the wave solver, as demonstrated in [5], allowing use of CFL numbers of 20-100+ (naturally with some loss of time accuracy as the time step size increases). Appropriate filtering techniques, perhaps using the artificial dissipation introduced in [6] or an adaptive variation thereof or perhaps using other standard techniques [31], could be applied to suppress numerical Cerenkov instabilities in simulations where they arise. Further, our potential-based formulation results in the uncoupling of the four components of the scalar and vector potential and with each wave equation having only one component of the charge density or current density appearing as a source, whereas the PSATD curl equations couple the six components of the electric and magnetic fields, with many components of the current density appearing in each equation (due to $\mathbf{k} \times \mathbf{J}$ terms). Hence, we expect that we can exploit a parallelism inherent in our choice of uncoupled governing equations in a way not possible with the coupled curl equations of the PSATD method.

In most PIC methods, further stability restrictions beyond the speed-of-light-based CFL restriction also apply, the most restrictive often being the need to resolve the electron plasma period. Our method, making use of explicit algorithms for advancing particles, is subject to such restriction. In problems where time scales much longer than the electron plasma period are of primary interest and dynamics on the scale of the electron plasma period can be safely underresolved - for instance, in certain problems in the study of ion dynamics - it is desirable to take a much larger time step than prescribed by the plasma period stability restriction. Since years ago [32, 33, 34, 35], and with a recent resurgence [36, 37, 38], it has been sought to develop implicit PIC algorithms that are not subject to the stability restriction based on the plasma period (or the cyclotron period). An ultimate challenge for our method would be synthesis with a suitable implicit particle integration scheme to achieve a practical fully implicit method, eliminating the stability restriction based on the plasma period as well as the field-based CFL restriction. However, that is beyond the scope of the present work.

We now outline the remainder of the paper. In Section 2, we describe the model equation sets used in this work. In Section 3, we describe the implicit wave solver used to evolve the fields. In Section 4, we discuss the details of the implementation of particles in the wave solver. In Section 5, we present the results of several numerical experiments, including two beam problems. In Section 6, we summarize our conclusions and present directions for further work. Additional information is presented in the Appendices. In Appendix A, we give the nondimensionalizations used in our test problems and asymptotic analyses that support the validity of our models, while in Appendix B, we present fully discrete Von Neumann analyses that prove the unconditional stability of the implicit wave solver used to compute the fields. In Appendix D, we present a time-staggered scheme that will be the basis of future development of our method. 


\section{Physical Models}

The self-consistent evolution of a collisionless, single species plasma is described by the Vlasov-Maxwell system, given in the SI system of units by

$$
\begin{array}{ccrl}
\frac{\partial f}{\partial t}+\mathbf{v} \cdot \nabla_{\mathbf{x}} f+\frac{q}{m}(\mathbf{E}+\mathbf{v} \times \mathbf{B}) \cdot \nabla_{\mathbf{v}} f & =0 \\
\epsilon_{0} \mu_{0} \frac{\partial \mathbf{E}}{\partial t}=\nabla \times \mathbf{B}-\mu_{0} \mathbf{J} & \frac{\partial \mathbf{B}}{\partial t}=-\nabla \times \mathbf{E} \\
\nabla \cdot \mathbf{E}=\rho / \varepsilon_{0} & \nabla \cdot \mathbf{B}=0 \\
\rho(\mathbf{x}, t)=q \int_{\mathbf{v}} f(\mathbf{x}, \mathbf{v}, t) d \mathbf{v}, & \mathbf{J}=q \int_{\mathbf{v}} \mathbf{v} f(\mathbf{x}, \mathbf{v}, t) d \mathbf{v}
\end{array}
$$

where $f(\mathbf{x}, \mathbf{v}, t)$ is the phase-space distribution function, $q$ is the charge and $m$ is the mass of a particle, $\mathbf{E}(\mathbf{x}, t)$ is the electric field, $\mathbf{B}(\mathbf{x}, t)$ is the magnetic field, $\rho(\mathbf{x}, t)$ is the charge density (and $\rho_{B}$ represents a static uniform background charge distribution), $\mathbf{J}(\mathbf{x}, t)$ is the current density, $\varepsilon_{0}$ is the electric permittivity of the vacuum, and $\mu_{0}$ is the magnetic permability of the vacuum. (Boldface variables are to stand for vector quantities, while nonboldface variables are to stand for scalar quantities.)

In the limit, in an appropriate sense, of $|\mathbf{v}| / c \rightarrow 0$ (where $c=1 / \sqrt{\varepsilon_{0} \mu_{0}}$ is the speed of light in vacuum) and in the absence of magnetic fields, the Vlasov-Maxwell system reduces to the Vlasov-Poisson system:

$$
\begin{gathered}
\frac{\partial f}{\partial t}+\mathbf{v} \cdot \nabla_{\mathbf{x}} f-\frac{q}{m} \nabla \Phi \cdot \nabla_{\mathbf{v}} f=0 \\
-\nabla^{2} \Phi=\rho / \varepsilon_{0}, \quad \rho(\mathbf{x}, t)=q \int_{\mathbf{v}} f(\mathbf{x}, \mathbf{v}, t) d \mathbf{v}
\end{gathered}
$$

The Vlasov-Maxwell and Vlasov-Poisson models have been widely studied, and many numerical methods have been developed to solve them, such as electrostatic and electromagnetic PIC methods [1, 2, 39, 40, 41, 18, 24], as well as Eulerian methods [42, 43, 44, 45, 46] and semi-Lagrangian methods [47, 48, 49, 50]. Instead of solving either of these systems directly, we seek to develop a semi-implicit approach for the fields based on a vector potential formulation. Under the Lorenz gauge condition,

$$
\frac{1}{c^{2}} \frac{\partial \Phi}{\partial t}+\nabla \cdot \mathbf{A}=0,
$$

Maxwell's equations reduce to uncoupled wave equations:

$$
\begin{aligned}
\frac{1}{c^{2}} \frac{\partial^{2} \Phi}{\partial t^{2}}-\nabla^{2} \Phi & =\rho / \varepsilon_{0} \\
\frac{1}{c^{2}} \frac{\partial^{2} \mathbf{A}}{\partial t^{2}}-\nabla^{2} \mathbf{A} & =\mu_{0} \mathbf{J}
\end{aligned}
$$


with $\mathbf{E}=-\nabla \Phi-\frac{\partial \mathbf{A}}{\partial t}$ and $\mathbf{B}=\nabla \times \mathbf{A}$ [51]. A similar potential-based approach was taken in [52], which used the Coulomb gauge condition $(\nabla \cdot \mathbf{A}=0)$, resulting in more a complicated set of coupled equations, which they solved using explicit finite difference methods. We choose to work insead in the Lorenz guage, as the resulting uncoupled wave equations are simpler to handle numerically.

In the electromagnetic case, we will solve these wave equations coupled into a particlein-cell method to constitute a solution of the usual Vlasov-Maxwell system. In the nonrelativistic, zero-magnetic field limit, we drop $\mathbf{A}$ and the corresponding wave equations and consider a quasi-electrostatic Vlasov-Wave model, which will agree with the electrostatic Vlasov-Poisson model when $|\mathbf{v}| / c<<1$. This condition frequently can be interpreted as $\omega_{p} L<<c$ for a relevant physical length scale $L$, where $\omega_{p}$ is the (electron) plasma frequency. In Appendix A, we present an asymptotic analysis that clarifies the relation between the Vlasov-Wave and Vlasov-Poisson systems as electrostatic limits of the Vlasov-Maxwell system. The resulting Vlasov-Wave system is as follows:

$$
\begin{gathered}
\frac{\partial f}{\partial t}+\mathbf{v} \cdot \nabla_{\mathbf{x}} f-\frac{q}{m} \nabla \Phi \cdot \nabla_{\mathbf{v}} f=0 \\
\frac{1}{c^{2}} \frac{\partial^{2} \Phi}{\partial t^{2}}-\nabla^{2} \Phi=\rho / \varepsilon_{0}, \quad \rho(\mathbf{x}, t)=q \int_{\mathbf{v}} f(\mathbf{x}, \mathbf{v}, t) d \mathbf{v}
\end{gathered}
$$

The purpose of this work is the numerical solution of (nondimensionalized forms of) both electromagnetic and electrostatic systems with PIC methods under a unified framework that avoid imposing a CFL stablity restriction related to the speed of light due to the presence of the wave equations for $\Phi$ and $\mathbf{A}$.

\section{Wave Solver Description}

\subsection{Origin and Mitigation of the CFL Restriction}

Consider the Cauchy problem for the wave equation:

$$
\begin{aligned}
u_{t t} & =c^{2} \nabla^{2} u \\
u(x, 0) & =g(x) \\
u_{t}(x, 0) & =h(x) .
\end{aligned}
$$

To see the origin of the CFL restriction in a typical explicit finite difference method, consider the semi-discrete scheme obtained by substituting the centered, second-order finite difference discretization of $u_{t t}$ into the wave equation. We obtain

$$
\frac{u(x, t+\Delta t)-2 u(x, t)+u(x, t-\Delta t)}{\Delta t^{2}}=c^{2} \nabla^{2} u(x, t) .
$$

Upon Fourier transforming in the spatial variable, we obtain

$$
\hat{u}(\xi, t+\Delta t)-2 \hat{u}(\xi, t)+\hat{u}(\xi, t-\Delta t)=-c^{2} \Delta t^{2}|\xi|^{2} \hat{u}(\xi, t)
$$


where $\xi$ is the spatial frequency. The problem is that a high-frequency perturbation of $u$ such as that introduced by truncation error - will be amplified by the symbol $-c^{2} \Delta t^{2}|\xi|^{2}$, resulting in instability. In a fully discrete method, the spatial frequencies are bounded by $2 / \Delta x$ (the Nyquist frequency), meaning that $c \Delta t / \Delta x$ must be sufficiently small to prevent amplification of high frequencies. In terms of semi-discrete Von Neumann analysis, consider substituting into 15 a semi-discrete solution of the form $\hat{u}(\xi, n \Delta t)=\lambda^{n}$ for some $\lambda \in \mathbb{C}$. We obtain a quadratic equation $\lambda^{2}-\left(2-z^{2}\right) \lambda+1=0$ and the stability condition $|\lambda| \leq 1$ for any root $\lambda$ of this equation, where $z=c \Delta t|\xi|$. It is easily verified from the quadratic formula that the roots $\lambda_{i}$ satisfy $\left|\lambda_{i}\right| \leq 1$ for $i=1,2$ if and only if $|z| \leq 2$, that is if and only if $c \Delta t \leq 2 /|\xi|$. For the 1D case, inserting the Nyquist frequency $2 / \Delta x$ into the upper bound gives the usual CFL stability restriction $c \Delta t \leq \Delta x$.

To avoid the CFL restriction while maintaining an explicit update equation, we modify the symbol of the Laplacian to bound it and prevent the amplification of high frequencies. Consider the one-dimensional case. We look for bounded rational approximations of $\xi^{2}$ near $\xi=0$. One option is

$$
\xi^{2} \approx \frac{\alpha^{2} \xi^{2}}{\xi^{2}+\alpha^{2}} \approx \xi^{2}\left(1-(\xi / \alpha)^{2}+\cdots\right)
$$

We can write this modified semi-discrete scheme in Fourier space as

$$
\hat{u}(\xi, t+\Delta t)-2 \hat{u}(\xi, t)+\hat{u}(\xi, t-\Delta t)=-c^{2} \Delta t^{2} \frac{\alpha^{2} \xi^{2}}{\xi^{2}+\alpha^{2}} \hat{u}(\xi, t)
$$

Choosing $\alpha=\alpha_{\beta}=\beta / c \Delta t$ for some properly chosen $\beta$, we can see that the modified symbol is uniformly bounded by $\beta^{2}$ for all $\Delta t$, so that choosing $\beta \leq 2$ will ensure stability, independently of $\Delta t$, and it turns out that $\beta=2$ gives optimal accuracy for stable schemes of this family $[6,5]$. Multiplication in Fourier space is equivalent to convolution in physical space, so transforming back into physical space gives a method based on convolution with the inverse Fourier transform of the modified symbol:

$$
u(x, t+\Delta t)-2 u(x, t)+u(x, t-\Delta t)=-\beta^{2} D[u](x, t)
$$

where $D[f](x)=\int\left(\delta\left(x-x^{\prime}\right)-\frac{\alpha}{2} e^{-\alpha\left|x-x^{\prime}\right|}\right) f\left(x^{\prime}\right) d x^{\prime}=f(x)-\frac{\alpha}{2} \int e^{-\alpha\left|x-x^{\prime}\right|} f\left(x^{\prime}\right) d x^{\prime}$.

As a general comment, this modification of the symbol of the Laplacian introduces error at high spatial frequencies, so that this technique may not be suitable for problems in which extremely accurate propagation of waves at high spatial frequencies (high wavenumber) is essential. However, in many problems of plasma physics, the physics is dominated by effects at low spatial frequencies, and in these problems this technique can find useful application.

A detailed discussion of numerical methods for the wave equation derived from the perspective of Fourier multipliers, including higher-order methods and the extension to multiple dimensions, can be found in [5]. In the following section, we give the derivation of two useful second-order numerical methods through alternative means, the Method of Lines Transpose, 
which also facilitates the extension of the methods to bounded domains and to multiple dimensions. Von Neumann analyses demonstrating the unconditional stability of the derived methods are presented in Appendix B.

\subsection{Method of Lines Transpose: Derivation of Second-Order Semi-Discrete Schemes}

In the Method of Lines Transpose for the solution of time-dependent PDEs, also known as Rothe's method [53], finite difference discretizations of time derivatives are substituted into the PDE, resulting in a boundary value problem (BVP) to be solved at each time step. In recent years, the MOLT has been applied as a numerical method to solve various time-dependent PDEs, with initial focus on parabolic equations [54, 55, 56, 57, 58]. The work [4] extended this approach to the second order wave equation, and is the basis of the present work. Further work extended this approach to higher dimensions through dimensional splitting [6] and to higher-order through the Fourier multiplier approach mentioned in the previous section [5]. Based on the work in $[4,6,5]$, we give here an overview of the derivation of two useful second-order schemes for the wave equation, their extension to multiple dimensions through dimensional splitting, and a fast numerical algorithm for the 1D problem. In Appendix B, we give semi-discrete Von Neumann analyses and dispersion relations for these semi-discrete schemes, along with a new proof that the fully discrete versions are unconditionally stable, in the sense of Von Neumann analysis.

\subsubsection{Dispersive Semi-Discrete Scheme}

As in the previous section, we substitute the following second-order centered discretization into the wave equation $\frac{1}{c^{2}} u_{t t}-\nabla^{2} u=f(x, t)$ :

$$
u_{t t}^{n}=\frac{u^{n+1}-2 u^{n}+u^{n-1}}{\Delta t^{2}}-\frac{\Delta t^{2}}{12} u_{t t t t}(x, \eta) .
$$

Instead of proceding by the Fourier multiplier approach as mentioned previously, we apply an averaging technique with similar results. We average the Laplacian term in time

$$
\nabla^{2} u^{n}=\frac{1}{4} \nabla^{2}\left(u^{n+1}+2 u^{n}+u^{n-1}\right)+O\left(\Delta t^{2}\right)
$$

and substitute into the wave equation. Defining $\alpha_{2}=2 /(c \Delta t)$ and manipulating gives the semi-discrete scheme

$$
\left(-\frac{1}{\alpha_{2}^{2}} \nabla^{2}+1\right)\left(u^{n+1}+2 u^{n}+u^{n-1}\right)=4 u^{n}+\frac{4}{\alpha_{2}^{2}} f\left(x, t^{n}\right)+O\left(\Delta t^{4}\right) .
$$

We call this the dispersive semi-discrete scheme, since all terms in the semi-discrete dispersion relation are real-valued (see Appendix B.1). 


\subsubsection{Diffusive Semi-Discrete Scheme}

We substitute the following backward difference formula (BDF) discretization:

$$
u_{t t}^{n+1}=\frac{2 u^{n+1}-5 u^{n}+4 u^{n-1}-u^{n-2}}{\Delta t^{2}}-\frac{11 \Delta t^{2}}{12} u_{t t t t}(x, \eta)
$$

into the wave equation $\frac{1}{c^{2}} u_{t t}-\nabla^{2} u=f(x, t)$.

Rearranging, defining $\alpha_{\sqrt{2}}=\sqrt{2} /(c \Delta t)$ and dividing by $\alpha_{\sqrt{2}}^{2}$ gives the semi-discrete scheme

$$
\left(-\frac{1}{\alpha^{2} \sqrt{2}} \nabla^{2}+1\right) u^{n+1}=\frac{1}{2}\left(5 u^{n}-4 u^{n-1}+u^{n-2}\right)+\frac{1}{\alpha^{2}} \underset{\sqrt{2}}{f} f\left(x, t^{n+1}\right)+O\left(\Delta t^{4}\right) .
$$

We call this the diffusive semi-discrete scheme, due to the presence of imaginary-valued terms in the semi-discrete dispersion relation (see Appendix B.1). The (slight) dissipation of this method proved useful in our PIC simulations as a means of reducing noise, a goal commonly pursued in PIC simulations through the use of filtering or by other means (see [3], and also [31] and the references within), and we use this scheme (rather than the dispersive scheme) in all numerical test problems in this work, and we will often simply write $\alpha$ instead

of $\alpha_{\beta}$ for the relevant values of $\beta=2, \sqrt{2}$. Future work will investigate the use of centered schemes such as the dispersive scheme given above and the higher-order accurate variations given in [5] in PIC simulations, including the use of artificial dissipation, when appropriate. In Appendix D we describe a time-staggered EM-PIC scheme using the dispersive wave solver, which is our closest analogue to the classical FDTD-Leapfrog EM-PIC method and which will be the basis of further development of our method.

\subsection{Solution of the Modified Helmholtz Equation}

As seen in the previous section, both the dispersive and diffusive semi-discrete schemes include an elliptic BVP to be solved at each time step. The resulting PDE is sometimes called the modified Helmholtz equation [56]. In contrast to the usual frequency-domain Helmholtz equation $\nabla^{2} u+\frac{\omega^{2}}{c^{2}} u=f$, the modified Helmholz equation has a nonoscillatory Green's function. The oscillation in the solution of the wave equation is supported by the presence of multiple time levels in the semi-discrete equations. Our solution strategy is to use the well-known technique of dimensional splitting [59] to reduce problems in multiple dimensions to problems in one dimension, to which we apply a fast integral solution method. The dimensionally-split integral solution naturally leads to unconditionally stable numerical schemes with computational cost and coding complexity comparable to explicit schemes.

\subsubsection{Dimensional Splitting}

For smooth $u(x, y)$, we have

$$
\left(-\frac{1}{\alpha^{2}}\left(\partial_{x x}+\partial_{y y}\right)+1\right) u=\left(-\frac{1}{\alpha^{2}} \partial_{x x}+1\right)\left(-\frac{1}{\alpha^{2}} \partial_{y y}+1\right) u-\frac{1}{\alpha^{4}} \partial_{x x y y} u
$$


So, to approximately solve $\left(-\frac{1}{\alpha^{2}}\left(\partial_{x x}+\partial_{y y}\right)+1\right) u=f$ we will instead solve

$$
\left(-\frac{1}{\alpha^{2}} \partial_{x x}+1\right)\left(-\frac{1}{\alpha^{2}} \partial_{y y}+1\right) u=f .
$$

where we have introduced the splitting error

$$
\frac{1}{\alpha^{4}} \partial_{x x y y} u=O\left(c^{4} \Delta t^{4}\right) .
$$

To solve

$$
\left(-\frac{1}{\alpha^{2}} \partial_{x x}+1\right)\left(-\frac{1}{\alpha^{2}} \partial_{y y}+1\right) u=f
$$

we define $w=\left(-\frac{1}{\alpha^{2}} \partial_{y y}+1\right) u$ and solve the following one-dimensional BVPs "line by line":

$$
\begin{aligned}
& \left(-\frac{1}{\alpha^{2}} \partial_{x x}+1\right) w(x, \cdot)=f(x, \cdot) \\
& \left(-\frac{1}{\alpha^{2}} \partial_{y y}+1\right) u(\cdot, y)=w(\cdot, y)
\end{aligned}
$$

where appropriate boundary conditions are supplied (see $[4,5])$. Since $w=u+O\left(c^{2} \Delta t^{2}\right)$, for second order accuracy it suffices to use the same boundary conditions for $w$ as $u$. Future work will investigate higher-order accurate boundary conditions. To facilitate the "line-by-line" solution, we discretize the domain with a uniform Cartesian grid.

\subsubsection{Integral Solution Method in 1D}

Consider the modified Helmholtz equation in one dimension, $\left(-\frac{1}{\alpha^{2}} \frac{d^{2}}{d x^{2}}+1\right) u=f(x)$ for $x \in \Omega=(a, b)$, with appropriate boundary conditions imposed at $x=a$ and $x=b$. We can write $u(x)=u^{P}(x)+u^{H}(x)$, where

$$
\begin{aligned}
u^{P}(x) & =\frac{\alpha}{2} \int_{a}^{b} f\left(x^{\prime}\right) e^{-\alpha\left|x-x^{\prime}\right|} d x^{\prime} \\
u^{H}(x) & =A e^{-\alpha(x-a)}+B e^{-\alpha(b-x)}
\end{aligned}
$$

are the particular and homogeneous solutions and where $A$ and $B$ depend on the boundary conditions imposed, as well as the values of $u^{P}(a)$ and $u^{P}(b)$ [4]. Our fast integral solver consists of a fast convolution algorithm for the evaluation of the particular solution $u^{P}$, along with appropriate algorithms for evaluating the homogeneous solution $u^{H}$, which can be viewed as boundary correction terms. For many common boundary conditions, the coefficients $A$ and $B$ for the boundary correction terms can be found by applying the given boundary conditions and solving a $2 \times 2$ system by hand for $A$ and $B$. For instance, in the case of homogeneous Dirichlet boundary conditions $u(a)=u(b)=0$ and defining $\gamma=e^{-\alpha(b-a)}$, 
we find

$$
\begin{aligned}
& A=\frac{\gamma u^{P}(b)-u^{P}(a)}{1-\gamma^{2}} \\
& B=\frac{\gamma u^{P}(a)-u^{P}(b)}{1-\gamma^{2}} .
\end{aligned}
$$

In the case of periodic boundary conditions, we find

$$
\begin{aligned}
& A=\frac{u^{P}(b)}{1-\gamma} \\
& B=\frac{u^{P}(a)}{1-\gamma} .
\end{aligned}
$$

With some further consideration, other boundary conditions can be derived, including outflow (absorbing) boundary conditions (for the underlying wave equation, based on one-way wave equations). For further details, see $[4,6,5]$.

\subsubsection{Fast Numerical Evaluation of the 1D Convolution Operator}

The convolution operator giving the particular solution can be decomposed as

$$
\begin{aligned}
I[f](x) & =\frac{\alpha}{2} \int_{-\infty}^{\infty} f\left(x^{\prime}\right) e^{-\alpha\left|x-x^{\prime}\right|} d x^{\prime} \\
& =\frac{\alpha}{2} \int_{-\infty}^{x} f\left(x^{\prime}\right) e^{-\alpha\left|x-x^{\prime}\right|} d x^{\prime}+\frac{\alpha}{2} \int_{x}^{\infty} f\left(x^{\prime}\right) e^{-\alpha\left|x-x^{\prime}\right|} d x^{\prime} \\
& =: I^{L}[f](x)+I^{R}[f](x)
\end{aligned}
$$

for a given function $f$. Meanwhile, we have the recursion relations

$$
\begin{gathered}
I^{L}[f](x)=e^{-\alpha \Delta x} I^{L}[f](x-\Delta x)+\frac{\alpha}{2} \int_{x-\Delta x}^{x} f\left(x^{\prime}\right) e^{-\alpha\left(x-x^{\prime}\right)} d x^{\prime} \\
I^{R}[f](x)=e^{-\alpha \Delta x} I^{R}[f](x+\Delta x)+\frac{\alpha}{2} \int_{x}^{x+\Delta x} f\left(x^{\prime}\right) e^{-\alpha\left(x^{\prime}-x\right)} d x^{\prime} .
\end{gathered}
$$

Based on these observations, we outline the fast algorithm for the numerical evaluation of this convolution operator on a uniform Cartesian grid developed in $[4,6,5]$. Consider the convolution operator applied to a function $f$ supported on the interval $(a, b)$, with the convolution also to be evaluated in $(a, b)$. The interval is discretized into $N$ equal subintervals of length $\Delta x=(b-a) / N$, with endpoints $x_{1}=a, x_{j+1}=x_{j}+\Delta x$ for $j=1, \ldots, N$. We denote $I_{j}=I[f]\left(x_{j}\right), I_{j}^{L}=I^{L}[f]\left(x_{j}\right)$ and $I_{j}^{R}=I^{R}[f]\left(x_{j}\right)$, as defined above. Further, we define the local integrals

$$
\begin{aligned}
& J_{j}^{L}=\frac{\alpha}{2} \int_{x_{j-1}}^{x_{j}} f\left(x^{\prime}\right) e^{-\alpha\left(x_{j}-x^{\prime}\right)} d x^{\prime} \quad j=2, \ldots, N+1 \\
& J_{j}^{R}=\frac{\alpha}{2} \int_{x_{j}}^{x_{j+1}} f\left(x^{\prime}\right) e^{-\alpha\left(x^{\prime}-x_{j}\right)} d x^{\prime} \quad j=1, \ldots, N .
\end{aligned}
$$


Suppose we have already computed these $J_{j}^{L}$ and $J_{j}^{R}$ (not to be confused with the current density). Setting $I_{1}^{L}=I_{N+1}^{R}=0$, we can then perform a recursive evaluation of the convolution integral by computing $I_{j+1}^{L}=e^{-\alpha \Delta x} I_{j}^{L}+J_{j+1}^{L}$ and $I_{N+1-j}^{R}=e^{-\alpha \Delta x} I_{N+1-j+1}^{R}+J_{N+1-j}^{R}$ for $j=1, \ldots N$. We then sum $I_{j}=I_{j}^{L}+I_{j}^{R}$ for each $j=1, . . N+1$.

The method for evaluating the local integrals $J_{j}^{L}$ and $J_{j}^{R}$ depends on the nature of the integrand. For the particle convolution integral, where the integrand is the sum of particle shape functions, we can analytically evaluate the local integral contributions of each particle. This is described in detail in Appendix C. For general integrands, and specifically for the terms involving $u$ on the right hand sides of the semi-discrete schemes, we numerically evaluate the local integrals with a quadrature rule. It is important to note that any given quadrature rule may or may not deliver an accurate and stable overall scheme for the wave equation. To achieve accuracy and stability, we use a quadrature rule found by analytically integrating against a Lagrange polynomial interpolant. For a quadratic interpolant, leading to second-order accurate quadrature in space, we obtain the following approximations

$$
\begin{aligned}
& J_{j}^{L} \approx P f\left(x_{j}\right)+Q f\left(x_{j-1}\right)+R\left(f\left(x_{j+1}\right)-2 f\left(x_{j}\right)+f\left(x_{j-1}\right)\right) \\
& J_{j}^{R} \approx \operatorname{Pf}\left(x_{j}\right)+Q f\left(x_{j+1}\right)+R\left(f\left(x_{j+1}\right)-2 f\left(x_{j}\right)+f\left(x_{j-1}\right)\right)
\end{aligned}
$$

where, defining $\nu=\alpha \Delta x$ and $d=e^{-\nu}$,

$$
\begin{aligned}
P & =1-\frac{1-d}{\nu} \\
Q & =-d+\frac{1-d}{\nu} \\
R & =\frac{1-d}{\nu^{2}}-\frac{1+d}{2 \nu}
\end{aligned}
$$

Higher-order spatial accuracy can be obtained by using higher-order accuracy quadrature rules, with the outcome of unconditional stability limiting the choice of quadrature rules. Further details can be found in $[4,5,6]$. In Appendix B.2, Von Neumann analyses are carried out for the fully discrete diffuse and dispersive schemes in one and multiple dimensions, and it is shown that they are both unconditionally stable.

Remark. We note that dimensional splitting approach taken here naturally admits tensor product grid refinement without need for further modification of the underlying wave solver (though the coupling with particles must be suitably modified in a PIC method). Other approaches to grid refinement, such as those reviewed in the introduction, could be adapted to our method, but this is left to future work. 


\section{Implementation of Particles}

\subsection{Particle Weighting Scheme}

In our PIC methods, the charge and current densities are represented as the sum of particle shape functions:

$$
\begin{aligned}
\rho(\mathbf{x}, t) & =\sum_{i=1}^{N_{p}} q_{i} S\left(\frac{\mathbf{x}-\mathbf{x}_{p, i}(t)}{\Delta x}\right) \\
\mathbf{J}(\mathbf{x}, t) & =\sum_{i=1}^{N_{p}} q_{i} \mathbf{v}_{p, i} S\left(\frac{\mathbf{x}-\mathbf{x}_{p, i}(t)}{\Delta x}\right)
\end{aligned}
$$

where $N_{p}$ is the number of particles, $\mathbf{x}_{p, i}(t)$ and $\mathbf{v}_{p, i}(t)$ are the position and velocity, respectively, of particle $i$ at time $t$, and $S(\mathbf{x})$ is a particle shape function. It should be emphasized that these are not physical particles, but rather macro- or superparticles that represent a discretization of the PDF [1]. We analytically evaluate the particle convolution integrals corresponding to these source terms with the algorithms described below.

\subsection{Method for Controlling Divergence Error}

It is well known that electromagnetic PIC methods which do not satisfy a discrete form of Gauss' law through their field solvers and charge and current weighting schemes will suffer severe numerical errors related to charge conservation [9]. We seek to develop staggered grid approaches to computing potentials and fields that satisfy discrete analogues of Gauss' law $\nabla \cdot \mathbf{E}=\rho / \epsilon_{0}$ and the identity $\nabla \cdot \mathbf{B}=0$. The divergence-free condition for $\mathbf{B}$ will be easily satisfied in general, as the magnetic field will be calculated as a finite difference curl of the vector potential. In order to numerically enforce Gauss' law, we seek to perform an elliptic divergence correction. Future work will consider the alternative of hyperbolic divergence cleaning [60].

We now give the mathematical underpinning of our elliptic divergence correction technique. The electric field is calculated as $\mathbf{E}=-\nabla \Phi-\frac{\partial \mathbf{A}}{\partial t}$. Then Gauss' law may be rewritten as:

$$
\begin{aligned}
\rho / \epsilon_{0} & =\nabla \cdot \mathbf{E} \\
& =\nabla \cdot\left(-\nabla \Phi-\frac{\partial \mathbf{A}}{\partial t}\right) \\
& =-\nabla^{2} \Phi-\frac{\partial(\nabla \cdot \mathbf{A})}{\partial t} .
\end{aligned}
$$

Thus, the scalar potential satisfies the Poisson equation

$$
-\nabla^{2} \Phi=\rho / \epsilon_{0}+\frac{\partial(\nabla \cdot \mathbf{A})}{\partial t}
$$

Our method is based on the observation that if this Poisson equation is suitably discretized and solved on a staggered grid to provide the scalar potential used in calculating the electric 
field, then the electric field will automatically satisfy a discrete form of Gauss' law. While the exact form of the staggered grid will depend on which components of the current density and the electric and magnetic fields are retained in a given model, our method guarantees exact discrete divergence relations independently of the charge and current weighting schemes used, of the nature of the solver used for $\mathbf{A}$, and of the gauge condition specified. Details about the specific staggered grids used are given in 5.2. It should be noted that this procedure is an analogue of the elliptic divergence correction techniques presented in [61,9], and also bears similarity to the method given in [52] to enforce the divergence relations when evolving the potentials in the Coulomb gauge. In order to impose outflow boundary conditions, we solve an auxiliary wave equation for $\Phi$ using our wave solver with outflow boundary conditions, which provides the boundary values for $\Phi$ in the Poisson solve.

\subsection{Particle Equations of Motion}

In our PIC methods, the approximation of the evolution of the Vlasov equation amounts to the integration of the equations of motion of the particles:

$$
\begin{aligned}
& \frac{d \mathbf{x}_{p, i}}{d t}=\mathbf{v}_{p, i}(t) \\
& \frac{d \mathbf{v}_{p, i}}{d t}=\mathbf{a}_{p, i}(t)
\end{aligned}
$$

where $\mathbf{a}_{p, i}(t)$ is the acceleration of particle $i$ at time $t$. To evolve the particle equations of motion, we obtain particle accelerations through the usual interpolation of fields from grid points to particle locations [1], and we use standard numerical methods, such as the explicit leapfrog method for electrostatic problems and the forward Euler method for electromagnetic problems (the implicit source term in the diffusive wave solver causes some difficulty in achieving higher-order accuracy in the integration of the particle equations of motion - for this reason the dispersive scheme and higher-order centered schemes given in [5] will be pursued in future work - but the accuracy achieved is sufficient for the numerical results in this work). Fields are calculated as finite differences of potentials on the grid. As these aspects are standard and not the focus of the present work, we do not elaborate further.

\subsection{Overview of the Fast Particle Convolution Algorithm}

We now give a brief overview of the algorithm used to evaulate the convolution integrals of source terms (43) and (44). A detailed description of the algorithm is given in Appendix C. The fast particle convolution algorithm is similar to the algorithm described in Section 3.3.3, proceeding by the computation of local integrals followed by a recursive sweep step. The local integral contributions of the particles are evaluated by analytic integration of the particle shape functions, in contrast to the local integral contributions of the time history terms, which are evaluated with numerical quadrature. Expressions for the local integral contributions of a particle with a piecewise linear shape function (hat function) are given in Appendix C. Corresponding expressions can also be obtained for higher-order particle shape functions. 


\subsection{Particle Boundary Conditions}

In dealing with boundaries, two types of considerations must be made. First, we must determine what to do in the integration of boundary particles, for which the support of the shape function extends outside of the domain. This will be dependent upon the type of boundary condition. For periodic boundary conditions, we can simply extend the particle shape function periodically and proceed to integrate. For Dirichlet boundary conditions, we extend the integration domain to include ghost points just beyond the boundary to which the boundary particles are weighted.

Second, we must ensure that the particle convolution integral is consistent with the boundary conditions on the wave function. This is easily handled through the usual boundary correction terms in one-dimension, and can be extended to the dimensionally-split multidimensional case.

\section{Numerical Results}

\subsection{Electrostatic Test Problems}

We first consider three standard periodic electrostatic test problems in 1D and 2D, then a 1D bounded plasma problem, the simulation of sheath formation. In the first three test problems, electrons are loaded from a perturbed initial distribution of the form

$$
f_{e}(x, v, t=0)=f_{e}(v)\left(1+\epsilon \sin \left(\frac{2 \pi x}{L_{x}}\right)\right)
$$

where $L_{x}$ is the length of the domain, $\epsilon$ is the amplitude of perturbation, and $f_{e}(v)$ is the initial velocity distribution. In the $2 \mathrm{D}$ case, simulations are taken to be uniform in the $y$ direction. We normalize quantities according to the nondimensionalization presented in the Section Appendix A.1. In particular, we normalize time quantities to the inverse plasma frequency $\omega_{p}^{-1}$. We will consider a periodic domain with a uniform neutralizing background charge, and further we set the speed of light $c=100$. In these problems, we see good performance even at large CFLs, since the physics is dominated by the low frequency spatial modes.

\subsubsection{Cold Plasma Langmuir Wave}

We consider a cold plasma Langmuir wave [1] with $f_{e}(v)=\delta(v)$. Electrons are perturbed away from a uniformly distributed, motionless state against a static, uniform neutralizing background charge distribution. The resulting separation of charge produces cold plasma oscillation. In the $1 \mathrm{D}$ simulation, we set $L_{x}=2 \pi$ and $\epsilon=0.1$. We use a 100 cell grid and take $\Delta t=0.1$, and we use $N_{p}=3600$ particles. In the $2 \mathrm{D}$ simulation, we set $L_{x}=L_{y}=2 \pi$ and the perturbation strength $\epsilon=0.1$. We use a $100 \times 100$ grid and again take $\Delta t=0.1$, and we use $N_{p}=360000$ particles. In both $1 \mathrm{D}$ and $2 \mathrm{D}$ cases, the CFL number used is $c \Delta t / \Delta x \approx 159$, much larger than what would be allowed by an explicit method. The oscillation in the potential energy is plotted and compared to the prediction of linear theory in Figure 1; we see that the plasma frequency is accurately reproduced. 


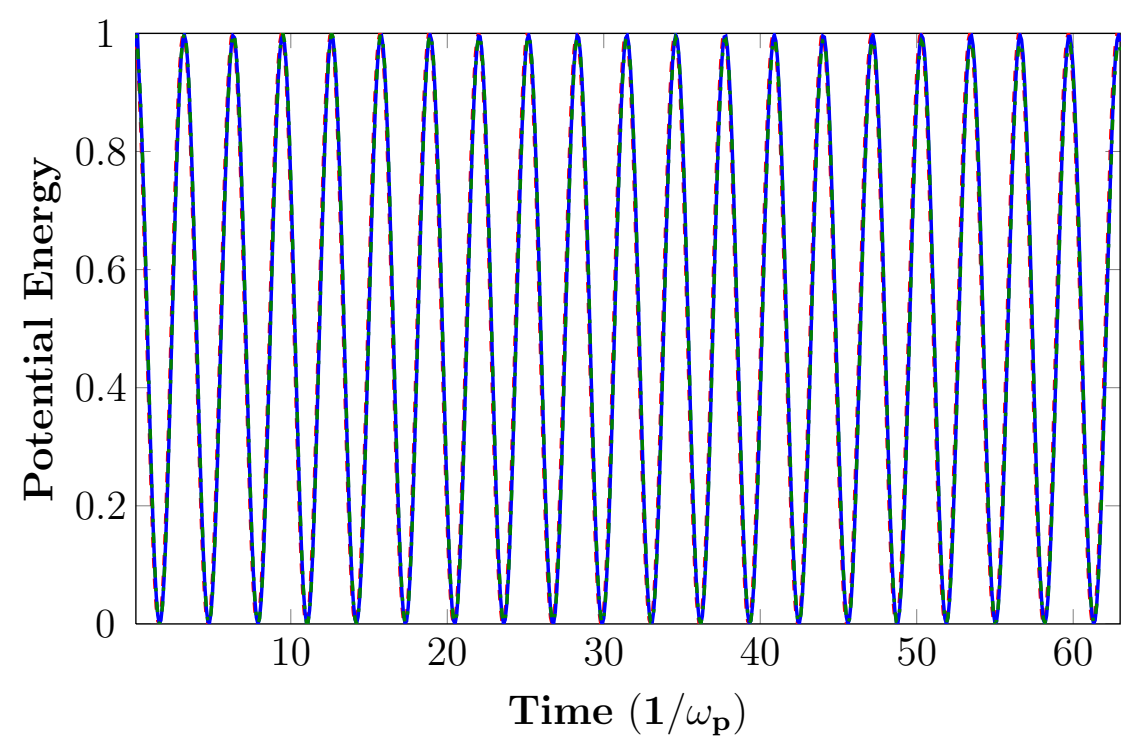

Figure 1: Potential energy in cold plasma oscillation. Green is the 1D numerical result, blue is the $2 \mathrm{D}$ numerical result, and red is the prediction of linear theory. We see the plasma frequency is accurately reproduced in our simulations.

\subsubsection{Two Stream Instability}

We consider the two stream instability with $f_{e}(v)=\delta\left(v-v_{\text {beam }}\right)+\delta\left(v+v_{\text {beam }}\right)$. Two counterstreaming beams of electrons are perturbed away from a uniformly distributed state against a static, uniform neutralizing background charge distribution. The beams interact and "roll up" in phase space, causing some of the particles' kinetic energy to be transformed into potential energy stored in the electric field. According to the dispersion relation for the two stream instability from linear theory [1], we have

$$
\omega^{4}-2 \omega^{2}\left(\omega_{p}^{2}+k^{2} v_{\text {beam }}^{2}\right)+k^{2} v_{\text {beam }}^{2}\left(k^{2} v_{\text {beam }}^{2}-2 \omega_{p}^{2}\right)=0
$$

which gives the greatest growth rate, $\gamma \approx 0.3535$, for $k \approx 3.06$. We therefore scale the domain to this value of $k$, and take $L_{x}=2 \pi / 3.06$. We take the beam velocity $v_{\text {beam }}=1$ and the perturbation strength $\epsilon=0.0005$. In our 1D simulation, we use a 100 cell grid with $\Delta t=0.1$, and we use $N_{p}=1000$ particles. In our 2 D simulation, we use a $100 \times 100$ grid with $\Delta t=0.1$, and we use $N_{p}=1000000$ particles. This results in a CFL number of $c \Delta t / \Delta x \approx 68$. We run the simulations for 1000 time steps. The growth of the $k=3.06$ mode of the electric field is shown in Figure 2 for the 1D and 2D cases, and agrees with the rate from linear theory. In the nonlinear saturation stage, we see a slight discrepancy between the 1D and $2 \mathrm{D}$ results, probably due to the accumulation of numerical error. We also show selected phase space plots in Figure 3, where we see the expected "rolling up" of the two beams, with some scattering of particles due to the limited resolution of the simulation. Liouville's theorem implies that the initial continuous curves in phase space should remain continuous curves later in time, however twisted and distorted they may become, which seeems to be violated by the appearance of such scattered particles. Such an apparent numerical violation 
of Liouville's theorem in a PIC simulation of the two stream instability was also observed in [62], which the authors of that work attributed to numerical noise pushing particles off of their true trajectories, with the onset of this noise coinciding with the onset of the exponential growth of the electric field energy. We therefore also present a corresponding plot of phase space at the final simulation time from a refined 1D simulation, with the grid and time step sizes decreased by a factor of 2 and the total number of particles increased by a factor of 400, which displays continuous particle distribution curves instead of the scattered particles of the initial simulation, indicating they are artifacts of poorly resolved features in phase space.

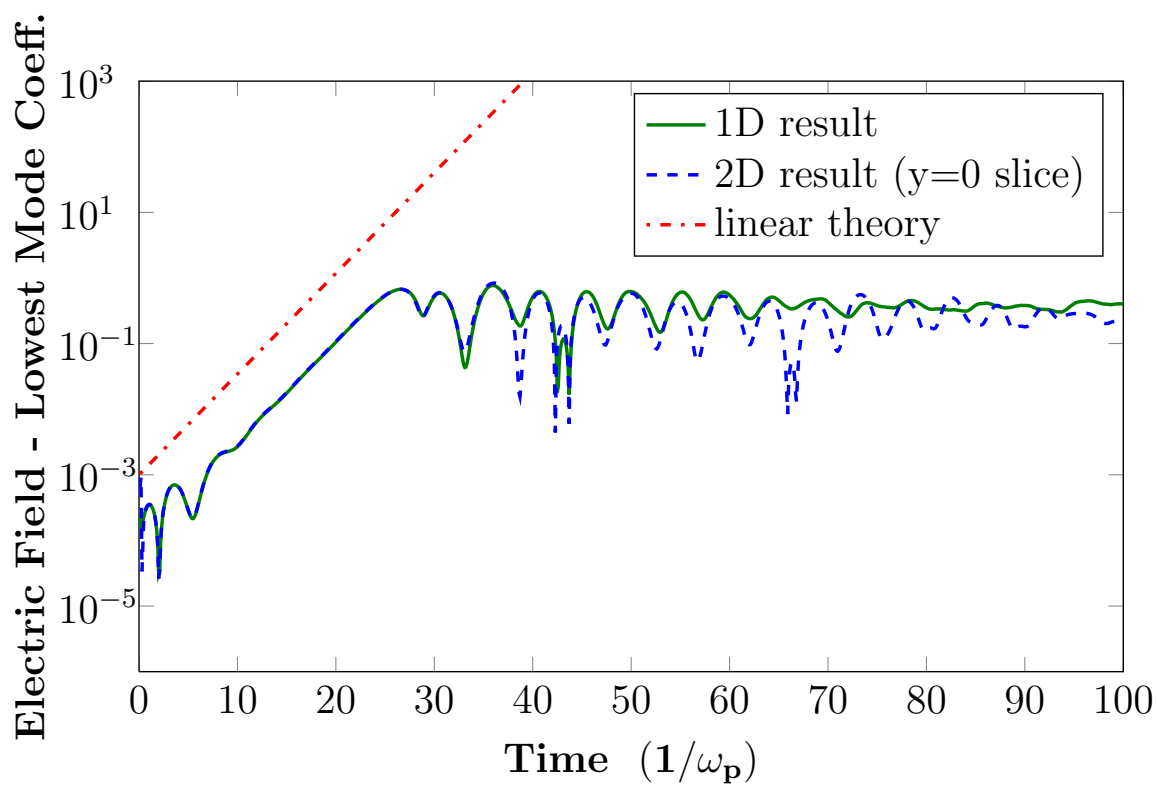

Figure 2: Growth of the mode with maximum growth rate in the two stream instability, corresponding to $k=3.06$. Green is the $1 \mathrm{D}$ numerical result, blue is the $2 \mathrm{D}$ numerical result (measured along the central $y=0$ slice), and red is the prediction of linear theory. We see the correct growth rate is reproduced in our simulations.

\subsubsection{Landau Damping}

We consider Landau damping of Langmuir waves in a warm plasma, with $f_{e}(v)$ taken to be Maxwellian. Warm electrons, following a Maxwellian velocity distribution, are perturbed away from a unifom distribution against a static, uniform neutralizing background charge distribution. Potential energy from the electric field is transformed into kinetic energy of particles. The dispersion relation from linear theory in this case gives a decay rate of $\gamma \approx$ 0.154 for the $k=0.5$ mode [1]. We take $L_{x}=4 \pi$, electron thermal velocity $v_{\text {therm }}=1$ and perturbation strength $\epsilon=0.1$. In our $1 \mathrm{D}$ simulation, we use a 100 cell grid and take $\Delta t=0.1$, and we use $N_{p}=1000000$ particles. In our $2 \mathrm{D}$ simulation, we use a $100 \times 100$ grid and take $\Delta t=0.1$, and we use $N_{p}=9000000$ particles. We run the simulations for 300 
time steps. The decay of the $k=0.5$ mode of the electric field in the $1 \mathrm{D}$ and $2 \mathrm{D}$ simulations is shown in Figure 4, and agrees with the rate from linear theory. As in the two stream instability example, there is a discrepancy between the 1D and 2D results at later times, again likely due to the accumulation of numerical errors.

\subsubsection{Sheath Formation in a Bounded 1D Plasma}

We present the simulation of sheath formation in a bounded 1D plasma, following the model described in [63]. In contrast to the previous problems, this simulation incorporates both mobile electrons and ions. Electrons and ions are initialized from Maxwellian distributions and uniformly spatially distributed in a bounded domain. The left boundary is a symmetry plane, and so we impose Neumann boundary conditions on the potential, and reflux boundary conditions on particles, as in [63]. The right boundary is a conductor that collects charged particles. When particles hit the right boundary, they are removed from the simulation. Since electrons have a higher average velocity than ions, they have a greater flux on the collector and become depleted near the right boundary, where the difference between ion and electron densities leads to a collector sheath region, where the potential changes from the interior value to the wall value, which has the effect of repelling electrons away from the right wall. Electrons and ions are replenished by a particle source region near the left boundary, where electrons and ions are injected uniformly in the region from a Maxwellian distribution at a fixed rate per time step. We take $m_{i} / m_{e}=100, T_{s r c, i} / T_{s r c, e}=1$, and we set $v_{t h, e}=1$, and set $L_{x}=20$ (in Debye lengths). We use a 100 cell grid and take $\Delta t=0.1$, which gives a CFL number of 50. We run our simulation for 8000 time steps, up to 3.6 thermal-ion transit times. In Figure 5 we see the result of the simulation. In Figure $5 \mathrm{a}$, we see the profile of the potential, which has the right qualitative features, including a collector sheath region that is several Debye lengths wide. In Figure 5b, we see the net electron and ion counts, along with the injection rate. The difference between the electron and ion counts reflects the difference between electron and ion densities in the collector sheath region.

\subsection{Electromagnetic Test Problems}

\subsubsection{Bennett Pinch Problem}

We present the application of our PIC method to the Bennett pinch [64], an effect related to the magnetic confinement of a beam of charged particles. A beam of charged particles induces a solenoidal magnetic field around the beam. Particles near the edge of the beam move orthogonally to these field lines at the beam drift velocity, causing the particles to be accelerated towards the center of the beam, in effect confining particles in the beam. An appropriate choice of parameters leads to a stationary steady state, uniform along the axis of the beam. A well-known magnetohydrodynamic (MHD) model of a stationary steady state gives explicit formulas for the beam density and the magnetic field [65], and provides a basis for the validation of our numerical method. Moreover, it is a first step toward applying our method to more physically interesting beam instability problems in three dimensions.

Our PIC simulation of the Bennett pinch is two-dimensional in physical space, and threedimensional in velocity space. The particle beam is considered uniform along the axis of the 
beam, which we take to be the $z$-direction, which reduces the physical dimensions to two. Electrons drift in the $z$-direction with a uniform average beam drift velocity $v_{b}$, and this motion induces a confining magnetic field with only $x$ - and $y$-components. A stationary ion background distribution enforces quasineutrality in the beam, with any separation of charge producing an electric field with only $x$ - and $y$-components acting as a restoring force. The electrons are assumed to follow a Maxwellian distribution with thermal velocity $v_{t h}$. We take $v_{b} / v_{t h}=100$ and $c / v_{t h}=1000$, where $c$ is the speed of light. The ions are considered cold $\left(T_{i}=0\right)$.

Since the beam drift velocity is taken to be much larger than the (transverse) thermal velocity, and further, the transverse velocities follow a Maxwellian distribution and so should not generate any net currents, we neglect the $x$ - and $y$-components of the current density (and so also of $\mathbf{A})$. In the true solution, $\frac{\partial \Phi}{\partial z}=0$ and $\frac{\partial A_{z}}{\partial t}=0$, so we neglect $E_{z}=-\frac{\partial \Phi}{\partial z}-\frac{\partial A_{z}}{\partial t}$. Hence, we actually only solve two wave equation, one for $A_{z}$, obtaining only transverse magnetic field components, $B_{x}=\frac{\partial A_{z}}{\partial y}$ and $B_{y}=-\frac{\partial A_{z}}{\partial x}$, and one for $\Phi$, obtaining only transverse electric field components $E_{x}=-\frac{\partial \Phi}{\partial x}$ and $E_{y}=-\frac{\partial \Phi}{\partial y}$. Thus, the Poisson equation satisfied by the scalar potential is $-\nabla^{2} \Phi=\rho / \epsilon_{0}$. We discretize our domain with a staggered grid, one cell of which is shown in Figure 6. The scalar potential is calculated from the standard 5-point finite difference Laplacian, and satisfies the equation

$$
-\frac{\Phi^{i+1, j}+\Phi^{i, j+1}+\Phi^{i-1, j}+\Phi^{i, j-1}-4 \Phi^{i, j}}{\Delta x^{2}}=\rho^{i, j} / \epsilon_{0} .
$$

The electric and magnetic fields are calculated on the staggered grid by finite differences as

$$
\begin{aligned}
& E_{x}^{i+1 / 2, j}=-\frac{\Phi^{i+1, j}-\Phi^{i, j}}{\Delta x} \\
& E_{y}^{i, j+1 / 2}=-\frac{\Phi^{i, j+1}-\Phi^{i, j}}{\Delta x} \\
& B_{x}^{i, j+1 / 2}=\frac{A_{z}^{i, j+1}-A_{z}^{i, j}}{\Delta x} \\
& B_{y}^{i+1 / 2, j}=-\frac{A_{z}^{i+1, j}-A_{z}^{i, j}}{\Delta x} .
\end{aligned}
$$

The electric field then satisifies the following discrete analogue of Gauss' law:

$$
\begin{aligned}
{[\nabla \cdot \mathbf{E}]^{i, j} } & =\frac{E_{x}^{i+1 / 2, j}-E_{x}^{i-1 / 2, j}}{\Delta x}+\frac{E_{y}^{i, j+1 / 2}-E_{y}^{i, j-1 / 2}}{\Delta x} \\
& =\frac{1}{\Delta x}\left(\left(-\frac{\Phi^{i+1, j}-\Phi^{i, j}}{\Delta x}\right)-\left(-\frac{\Phi^{i, j}-\Phi^{i-1, j}}{\Delta x}\right)+\right. \\
& \left.+\left(-\frac{\Phi^{i, j+1}-\Phi^{i, j}}{\Delta x}\right)-\left(-\frac{\Phi^{i, j}-\Phi^{i, j-1}}{\Delta x}\right)\right) \\
& =-\frac{\Phi^{i+1, j}+\Phi^{i, j+1}+\Phi^{i-1, j}+\Phi^{i, j-1}-4 \Phi^{i, j}}{\Delta x^{2}} \\
& =\rho^{i, j} / \epsilon_{0} .
\end{aligned}
$$


The magnetic field satisfies the following discrete analogue of the divergence free condition:

$$
\begin{aligned}
{[\nabla \cdot \mathbf{B}]^{i+1 / 2, j+1 / 2} } & =\frac{B_{x}^{i+1, j+1 / 2}-B_{x}^{i, j+1 / 2}}{\Delta x}+\frac{B_{y}^{i+1 / 2, j+1}-B_{y}^{i+1 / 2, j}}{\Delta x} \\
& =\frac{1}{\Delta x}\left(\left(\frac{A_{z}^{i+1, j+1}-A_{z}^{i+1, j}}{\Delta x}\right)-\left(\frac{A_{z}^{i, j+1}-A_{z}^{i, j}}{\Delta x}\right)+\right. \\
& \left.+\left(-\frac{A_{z}^{i+1, j+1}-A_{z}^{i, j+1}}{\Delta x}\right)-\left(-\frac{A_{z}^{i+1, j}-A_{z}^{i, j}}{\Delta x}\right)\right) \\
& =0 .
\end{aligned}
$$

All computational boundaries in this problem are outflow boundaries. In order to supply the finite difference Poisson solver with suitable boundary values, the wave solver is applied with outflow boundaries conditions to evolve the wave potential $\Phi_{W}$ alongside the Poisson potential $\Phi$. The boundary values from $\Phi_{W}$ are then supplied to the Poisson solver to use in calculating $\Phi$. Once the wave solver reaches steady state, $\Phi_{W}$ and $\Phi$ differ only by $0.1 \%$ relative error, however, the wave potential gives a discrete electric field with divergence error on the order of $10^{-3}$, while the Poisson potential gives a discrete electric field with divergence error on the order of machine epsilon $10^{-16}$.

Like in the other test problems, we use the diffusive version of wave solver. Particle velocities in all three directions are updated with the nonrelativistic Boris push [1]. Particles are initialized according to the MHD steady state (according to the theoretical spatial density profile and the corresponding Maxwellian distribution in velocity space) and held fixed while the field solver is stepped to an approximate steady state, after which the particle push is turned on. The simulation is run to a final time of $R_{b} /\left(2 v_{t h}\right)$ (plus startup time), where $R_{b}$ is an effective beam radius and $v_{t h}$ is the thermal velocity, at which time there would be substantial spreading of the beam in absence of the confinement effect. We choose $R_{b}$ such that $99 \%$ of the particles in the theoretical beam are within this radius. In loading particles, the beam is cut off at radius $R_{b}$ (no particles are loaded outside of this radius). The computational domain is taken to be a $4 R_{b} \times 4 R_{b}$ square centered on the beam axis. The computational domain is truncated with outflow boundary conditions. Particles exiting the boundary of the computational domain are reinjected into the beam to maintain constant total current. However, since most particles should be confined within the beam, such boundary crossings should be rare.

Numerical results for the Bennett pinch are given in Figure 7. In order to resolve large gradients near the center of the beam, we use a 500-cell by 500-cell grid and a CFL number of $c \Delta t / \Delta x=3$ (except in Figure $7 \mathrm{c}$ as noted) and 500,000 electron particles. Final numerical solutions are shown at the final time, after 334 start up time steps and 20,834 PIC time steps. (The final time is approx. 22 plasma periods, and the diameter of the beam is approx. 280 Debye lengths.) In Figure 7a, we see good agreement between the numerical electron density and MHD theory. The inset zoomed portion shows a slight discrepency at the peak of the beam, due to statistical fluctuation caused by the finite number of particles. In Figure $7 \mathrm{~b}$, we see the time histories of the potential energy, calculated as $\sum_{j} \frac{\Delta x \Delta y}{2}\left[\frac{1}{\mu_{R}}\left(B_{x, j}^{2}+B_{y, j}^{2}\right)+\varepsilon_{R}\left(E_{x, j}^{2}+E_{y, j}^{2}\right)\right]$ where the sum is over grid points $j$ 
(with $\varepsilon_{R}$ and $\mu_{R}$ defined as in Section Appendix A.2), and the kinetic energy, calculated as $\sum_{i} \frac{1}{2} m_{i}\left(v_{x, i}^{2}+v_{y, i}^{2}+\left(v_{x, i}-v_{b}\right)^{2}\right)$ where the sum is over electron particles $i$. We see good energy conservation, despite the slight dissipation of the diffusive scheme. The initial spike in the potential energy is the result of transient waves, arising due to the beam turning on, and flowing out of the domain as the solution is stepped to a steady state. In Figure 7c, we see the result of refinement in $\Delta t$, keeping $\Delta x$ fixed, showing a profile of the azimuthal magnetic field $B_{\theta}$ along the central $y=0$ slice for CFL numbers of 3, 10 and 20, along with MHD theory. We observe approximate second-order convergence in $\Delta t$, as expected (a more robust convergence study is confounded by the slow convergence in particle number in PIC methods). Outside of the beam radius $R_{b}=1$, there is error associated with the finite cut-off radius of the beam (the theoretical beam density decays only algebraically). In Figure $7 \mathrm{~d}$, we see the numerical error of the azimuthal magnetic field $B_{\theta}$ (with a CFL number of of 3) normalized by the peak value of the magnetic field, and we see that there is a geometric pattern to the numerical error, characteristic of the dimensionally-split method. In addition to this splitting error, the total error is contributed to by errors associated with the spatial quadrature and the finite differences used to calculate the magnetic field (likely contributing to the large error at the center of the beam due to large gradients there) and with the finite beam cut-off radius and the outflow boundary condition (contributing most strongly near the boundary of the computational domain). These results show that our method can indeed simulate a basic electromagnetic plasma phenomenon with a CFL number larger than what is allowed by typical explicit schemes. The CFL number used in this problem is limited by the accuracy of the second-order wave solver. A higher-order wave solver, such as those in [5], would allow for a larger usable time step size, and will be the subject of further investigation.

\subsubsection{Mardahl Beam Problem}

We apply our method to the beam problem proposed in [9] as a diagnostic for the effects of divergence error. Particles are injected into the domain, which is a box with PEC walls, from the left wall, travel across the domain and are removed from the simulation as they hit the right wall. Parameters are chosen such that the beam should pass through the domain unperturbed.

In the Mardahl beam problem, we have currents in the plane of simulation only, and so we retain the $x$ - and $y$-components of the vector potential, $A_{x}$ and $A_{y}$, along with the scalar potential $\Phi$ in the model. We retain the electric field components $E_{x}=-\frac{\partial \Phi}{\partial x}-\frac{\partial A_{x}}{\partial t}$ and $E_{y}=-\frac{\partial \Phi}{\partial y}-\frac{\partial A_{y}}{\partial t}$ and the magnetic field component $B_{z}=\frac{\partial A_{y}}{\partial x}-\frac{\partial A_{x}}{\partial y}$. The Poisson equation satisfied by the scalar potential is

$$
-\nabla^{2} \Phi=\rho / \epsilon_{0}+\frac{\partial}{\partial t}\left(\frac{\partial A_{x}}{\partial x}+\frac{\partial A_{y}}{\partial y}\right)
$$

We discretize our domain with a staggered grid, one cell of which is shown in Figure 8. Denoting by $D_{\Delta t}$ a (linear) finite difference discretization of the time derivative operator 
$\partial / \partial t$, the scalar potential satisfies the equation

$$
\begin{aligned}
-\frac{\Phi^{i+1, j}+\Phi^{i, j+1}+\Phi^{i-1, j}+\Phi^{i, j-1}-4 \Phi^{i, j}}{\Delta x^{2}} & =\rho^{i, j} / \epsilon_{0}+ \\
& D_{\Delta t}\left(\frac{A_{x}^{i+1 / 2, j}-A_{x}^{i-1 / 2, j}}{\Delta x}+\frac{A_{y}^{i, j+1 / 2}-A_{y}^{i, j-1 / 2}}{\Delta x}\right) .
\end{aligned}
$$

The electric and magnetic fields are calculated on the staggered grid by finite differences as

$$
\begin{aligned}
E_{x}^{i+1 / 2, j} & =-\frac{\Phi^{i+1, j}-\Phi^{i, j}}{\Delta x}-D_{\Delta t}\left(A_{x}^{i+1 / 2, j}\right) \\
E_{y}^{i, j+1 / 2} & =-\frac{\Phi^{i, j+1}-\Phi^{i, j}}{\Delta y}-D_{\Delta t}\left(A_{y}^{i, j+1 / 2}\right) \\
B_{z}^{i+1 / 2, j+1 / 2} & =\frac{A_{y}^{i+1, j}-A_{y}^{i, j}}{\Delta x}-\frac{A_{x}^{i, j+1}-A_{x}^{i, j}}{\Delta y}
\end{aligned}
$$

The electric field then satisifies the following discrete analogue of Gauss' law:

$$
\begin{aligned}
{[\nabla \cdot \mathbf{E}]^{i, j} } & =\frac{E_{x}^{i+1 / 2, j}-E_{x}^{i-1 / 2, j}}{\Delta x}+\frac{E_{y}^{i, j+1 / 2}-E_{y}^{i, j-1 / 2}}{\Delta y} \\
& =\frac{1}{\Delta x}\left(\left(-\frac{\Phi^{i+1, j}-\Phi^{i, j}}{\Delta x}-D_{\Delta t}\left(A_{x}^{i+1 / 2, j}\right)\right)-\left(-\frac{\Phi^{i, j}-\Phi^{i-1, j}}{\Delta x}-D_{\Delta t}\left(A_{x}^{i-1 / 2, j}\right)\right)+\right. \\
& \left.+\left(-\frac{\Phi^{i, j+1}-\Phi^{i, j}}{\Delta x}-D_{\Delta t}\left(A_{y}^{i, j+1 / 2}\right)\right)-\left(-\frac{\Phi^{i, j}-\Phi^{i, j-1}}{\Delta x}-D_{\Delta t}\left(A_{y}^{i, j-1 / 2}\right)\right)\right) \\
& =-\frac{\Phi^{i+1, j}+\Phi^{i, j+1}+\Phi^{i-1, j}+\Phi^{i, j-1}-4 \Phi^{i, j}}{\Delta x^{2}}- \\
& D_{\Delta t}\left(\frac{A_{x}^{i+1 / 2, j}-A_{x}^{i-1 / 2, j}}{\Delta x}+\frac{A_{y}^{i, j+1 / 2}-A_{x}^{i, j-1 / 2}}{\Delta x}\right) \\
& =\rho^{i, j} / \epsilon_{0} .
\end{aligned}
$$

where we have used the linearity of $D_{\Delta t}$.

Numerical results for this problem are given in Figure 9, using a $64 \times 64$ grid and a CFL of 1 . We see the expected distortion of the beam in the case when the divergence error is not controlled through the elliptic correction, whereas the beam passes through the domain unperturbed, as expected, when using the Poisson-based potential.

\section{Conclusions and Future Work}

In this work, we have described a PIC method that uses an unconditionally stable wave equation solver to eliminate the CFL restriction on the ratio of the time step size to the spatial step size, typical of explicit methods, while retaining computational cost $\left(O\left(N+N_{p}\right)\right.$ for 
$N$ grid points and $N_{p}$ particles) and code complexity comparable to such explicit methods. Our numerical results show that we can apply our method to problems of plasma physics using a time step size larger than what would be allowed by a typical explicit field solver. We have seen that the usable time step size can be limited by the numerical accuracy of the method, especially when there are large gradients (short-wavelength/high-frequency content) in the solution. In future work, we will investigate the use of higher-order methods in our PIC method in order to increase the maximum usable time step size following the ideas presented in [5], and we will make use of the implicit wave solvers ability to handle complex boundary geometries without the use of a staircasing approximation, as demonstrated in [6]. Alternative means of controlling divergence error will also be considered, including hyperbolic divergence cleaning as well as modified Esirpekov current weighting schemes [30] (whose derivation may be facilitated by use of analytical relations for the convolution integral). Further, we will investigate grid refinement and domain decomposition schemes to support the development of a fully-featured, parallel High Performance Computing (HPC) implementaion of our method. It remains to demonstrate that our PIC method can provide some benefit in typical PIC simulations with physically relevant boundary conditions (in computational accuracy or efficiency, or in the ease or flexibility of implementation) over widely-used existing explicit methods and other newly proposed methods. The numerical results shown in this paper were produced by Matlab codes, developed in an ad hoc fashion and intended as a proof of concept rather than a source of honest timing data, so that the authors defer making such comparisons to future work presenting an HPC implementation. However, initial numerical experiments with the implicit wave solver applied in a small (serial) test code to a scalar wave equation indicate that it is faster (in terms of time to solution) than an explicit finite difference method if a CFL number of at least 1.5 is used in the implicit wave solver, while a smaller CFL number of less than 1 is used in the explicit finite difference method. Hence, we expect that we could achieve an increase in computational efficiency with our method over explicit methods if we are able to use a large enough time step. This further motivates the development of higher-order accurate versions of our method, which markedly improve the wave propagation characteristics of the implicit wave solver and would allow for the use of a larger time step size [5]. By drawing from the rich literature on the issues confronting existing PIC simulation methods (including especially the works reviewed in the introduction), the authors believe that the method presented in this paper could provide a framework to address the development of stable schemes that are higher-order accurate in time and space, the accurate treatment of complex boundary geometries, the implementation of grid refinement and domain decomposition schemes to enable efficient parallel computation, and the removal of time and grid step size restrictions through the development of implicit particle models and hybrid kinetic-fluid PIC simulation techniques appropriate to specific appliations $[66,67,17,68,33,35,69,70,71,72,73,74,75,76,77,78,79,38,80]$, in order to advance plasma simulation capabilities beyond their present state. 


\section{Appendix A. Nondimensionalization and Asymptotic Analysis}

We provide the normalizations used in the test problems presented in this work in both electrostatic and electromagnetic cases. In the electrostatic case, we argue by formal asymptotic analysis and classical solution formulas that the Vlasov-Wave system agrees with the Vlasov-Poisson system in the relevant scaling limit.

\section{Appendix A.1. Electrostatic Case}

Here we give the normalization used for the electrostatic test problems in Section 5.1. Consider the following change of variables:

$$
\tilde{f}=F f, \quad \tilde{t}=T t, \quad \tilde{\mathbf{x}}=L \mathbf{x}, \quad \tilde{\mathbf{v}}=V \mathbf{v}, \quad \tilde{\rho}=q N \rho, \quad \tilde{\Phi}=\Phi_{0} \Phi
$$

applied to the system

$$
\begin{gathered}
\frac{\partial \tilde{f}}{\partial \tilde{t}}+\tilde{\mathbf{v}} \cdot \nabla_{\tilde{\mathbf{x}}} \tilde{f}-\frac{q}{m} \nabla \tilde{\Phi} \cdot \nabla_{\tilde{\mathbf{v}}} \tilde{f}=0 \\
\frac{1}{c^{2}} \frac{\partial^{2} \tilde{\Phi}}{\partial \tilde{t}^{2}}-\nabla^{2} \tilde{\Phi}=\tilde{\rho} / \varepsilon_{0}, \quad \tilde{\rho}(\tilde{\mathbf{x}}, \tilde{t})=q \int_{\tilde{\mathbf{v}}} \tilde{f}(\tilde{\mathbf{x}}, \tilde{\mathbf{v}}, \tilde{t}) d \tilde{\mathbf{v}}
\end{gathered}
$$

Assuming the scalings,

$$
V=\frac{L}{T}, \quad T=\sqrt{\frac{m \varepsilon_{0}}{N q^{2}}}=\omega_{p}^{-1}, \quad \Phi_{0}=\frac{q N L^{2}}{\varepsilon_{0}}, \quad F=\frac{N^{\frac{2-d}{d}}\left(\varepsilon_{0} m\right)^{d / 2}}{q^{d} L^{d}}
$$

which are the natural scalings in the electrostatic limit, we obtain:

$$
\begin{gathered}
\frac{\partial f}{\partial t}+\mathbf{v} \cdot \nabla_{\mathbf{x}} f-\nabla \Phi \cdot \nabla_{\mathbf{v}} f=0 \\
\epsilon^{2} \frac{\partial^{2} \Phi}{\partial t^{2}}-\nabla^{2} \Phi=\rho, \quad \rho(\mathbf{x}, t)=\int_{\mathbf{v}} f(\mathbf{x}, \mathbf{v}, t) d \mathbf{v}
\end{gathered}
$$

where $\epsilon=\frac{L}{c T}=\frac{V}{c}$ (not to be confused with the electric permittivity $\varepsilon_{0}$ ). Note that $1 / \epsilon$ is the speed of propagation of waves in the potential in this normalization, and becomes large when $\epsilon$ is small, that is, when the characteristic particle velocities are small compared to the speed of light.

Assume the following formal asymptotic expansions:

$$
\begin{aligned}
f & =f_{0}+\epsilon f_{1}+\epsilon^{2} f_{2}+\cdots \\
\rho & =\int_{\mathbf{v}} f d \mathbf{v} \\
& =\int_{\mathbf{v}} f_{0} d \mathbf{v}+\epsilon \int_{\mathbf{v}} f_{1} d \mathbf{v}+\epsilon^{2} \int_{\mathbf{v}} f_{2} d \mathbf{v}+\cdots \\
& =\rho_{0}+\epsilon \rho_{1}+\epsilon^{2} \rho_{2}+\cdots \\
\Phi & =\Phi_{0}+\epsilon \Phi_{1}+\epsilon^{2} \Phi_{2}+\cdots
\end{aligned}
$$


Collecting in orders of $\epsilon$ :

$$
\begin{aligned}
& O(1): \quad \frac{\partial f_{0}}{\partial t}+\mathbf{v} \cdot \nabla_{\mathbf{x}} f_{0}-\nabla \Phi_{0} \cdot \nabla_{\mathbf{v}} f_{0}=0 \\
& -\nabla^{2} \Phi_{0}=\rho_{0}, \quad \rho_{0}=\int_{\mathbf{v}} f_{0} d \mathbf{v} \\
& O(\epsilon): \quad \frac{\partial f_{1}}{\partial t}+\mathbf{v} \cdot \nabla_{\mathbf{x}} f_{1}-\nabla \Phi_{0} \cdot \nabla_{\mathbf{v}} f_{1}-\nabla \Phi_{1} \cdot \nabla_{\mathbf{v}} f_{0}=0 \\
& -\nabla^{2} \Phi_{1}=\rho_{1}, \quad \rho_{1}=\int_{\mathbf{v}} f_{1} d \mathbf{v} \\
& O\left(\epsilon^{k}\right), \quad k \geq 2: \\
& \frac{\partial f_{k}}{\partial t}+\mathbf{v} \cdot \nabla_{\mathbf{x}} f_{k}-\sum_{j=0}^{k} \nabla \Phi_{j} \cdot \nabla_{\mathbf{v}} f_{k-j}=0 \\
& -\nabla^{2} \Phi_{k}=\rho_{k}-\frac{\partial^{2} \Phi_{k-2}}{\partial t^{2}}, \quad \rho_{k}=\int_{\mathbf{v}} f_{k} d \mathbf{v} .
\end{aligned}
$$

We note that the leading order is precisely the Vlasov-Poisson system (nondimensionalized under the same scalings). This formal computation suggests that our model will agree with the electrostatic model to $O(\epsilon=V / c)$ when particle velocities are small compared to the speed of light. This can be considered as a consequence of the strong Huygens' principle in odd spatial dimensions, and of a weaker decay property that holds in even spatial dimensions, which can be deduced from classical solution formulas [81, 82]. Consider the Cauchy problem,

$$
\begin{array}{rlrl}
\frac{1}{c^{2}} \frac{\partial^{2} u}{\partial t^{2}}-\nabla^{2} u & =f(x, t) & & (x, t) \in \mathbb{R}^{d} \times(0, \infty) \\
u(x, 0) & =0 & x & \in \mathbb{R}^{d} \\
u_{t}(x, 0) & =0 & x & \in \mathbb{R}^{d}
\end{array}
$$

for $d=2,3$ and $f$ sufficiently smooth with compact support, for which classical explicit solution formulas exist. We consider the case of $f$ having compact support in $B(0, R) \times$ $(0, T) \subset \mathbb{R}^{d} \times(0, \infty)$, where $B(0, R)=\left\{x \in \mathbb{R}^{d} \mid \sum_{j=1}^{d} x_{j}^{2}<R^{2}\right\}$. Classical solution formulas imply that for $x \in B(0, R)$ and $t>T+2 R / c T$, we have

$$
\begin{array}{lrl}
u(x, t) & =O\left((c t)^{-1}\right) & d=2 \\
u(x, t) & =0 & d=3 .
\end{array}
$$

As a generalization of this, for any sufficiently smooth $f(\cdot, t)$ supported in $B(0, R)$ for all $t>0$ with $f(\cdot, t)=f_{T}(\cdot)$ for all $t>T$, it is again easily argued that for $x \in B(0, R)$ and $t>T+2 R / c T$, we have

$$
\begin{array}{ll}
u(x, t)=u_{P}^{2}(x)+O\left((c t)^{-1}\right) & d=2 \\
u(x, t)=u_{P}^{3}(x) & d=3
\end{array}
$$


where $u_{P}^{d}(x)$ is the classical integral solution of the Poisson equation $-\nabla^{2} u_{P}^{d}=f_{T}$ in dimension $d$.

The convergence of solutions to the Vlasov-Maxwell system to those of the Vlasov-Poisson system has been rigorously considered in works such as $[83,84]$. It may be possible to apply similar techniques to rigorously study the convergence of solutions of our Vlasov-Wave model to those of the Vlasov-Poisson system, but this is outside of the scope of the present work.

\section{Appendix A.2. Electromagnetic Case}

Here we give the normalization used for the electromagnetic test problem in Section 5.2. Consider the following change of variables:

$$
\begin{aligned}
& \tilde{f}=F f, \quad \tilde{t}=T t, \quad \tilde{\mathbf{x}}=L \mathbf{x}, \quad \tilde{\mathbf{v}}=V \mathbf{v} \\
& \tilde{\rho}=q N \rho, \quad \tilde{J}_{z}=q V N J_{z} \\
& \tilde{\Phi}=\Phi_{0} \Phi, \quad \tilde{\mathbf{A}}=A_{0} A_{z}
\end{aligned}
$$

applied to the system

$$
\begin{array}{rr}
\frac{\partial \tilde{f}}{\partial \tilde{t}}+\tilde{\mathbf{v}} \cdot \nabla_{\tilde{\mathbf{x}}} \tilde{f}+\frac{q}{m}\left(-\nabla_{\tilde{\mathbf{x}}} \tilde{\Phi}-\tilde{\mathbf{v}} \times\left(\nabla_{\tilde{\mathbf{x}}} \times\left(0,0, \tilde{A}_{z}\right)\right)\right) \cdot \nabla_{\tilde{\mathbf{v}}} \tilde{f}=0 \\
\frac{1}{c^{2}} \frac{\partial^{2} \tilde{\Phi}}{\partial \tilde{t}^{2}}-\nabla^{2} \tilde{\Phi}=\tilde{\rho} / \varepsilon_{0}, & \tilde{\rho}(\tilde{\mathbf{x}}, \tilde{t})=q \int_{\tilde{\mathbf{v}}} \tilde{f}(\tilde{\mathbf{x}}, \tilde{\mathbf{v}}, \tilde{t}) d \tilde{\mathbf{v}} \\
\frac{1}{c^{2}} \frac{\partial^{2} \tilde{A}_{z}}{\partial \tilde{t}^{2}}-\nabla^{2} \tilde{A}_{z}=\mu_{0} \tilde{J}_{z}, & \tilde{J}_{z}(\tilde{\mathbf{x}}, \tilde{t})=q \int_{\tilde{\mathbf{v}}} \tilde{v}_{z} \tilde{f}(\tilde{\mathbf{x}}, \tilde{\mathbf{v}}, \tilde{t}) d \tilde{\mathbf{v}}
\end{array}
$$

Assuming the scalings,

$$
\begin{aligned}
& V=\frac{L}{T}, \quad T=\sqrt{\frac{m \varepsilon_{0}}{N q^{2}}}=\omega_{p}^{-1}, \\
& \Phi_{0}=\frac{q N L^{2} \varepsilon_{R}}{\varepsilon_{0}}, \quad A_{0}=\frac{\mu_{0} V q N L^{2}}{\mu_{R}}, \quad \varepsilon_{R} \mu_{R}=V^{2} / c^{2}, \\
& F=\frac{N^{\frac{2-d}{d}}\left(\varepsilon_{0} m\right)^{d / 2}}{q^{d} L^{d}}
\end{aligned}
$$

where $\varepsilon_{R}$ and $\mu_{R}$ are dimensionless parameters introduced to enforce the Lorenz gauge condition, we obtain:

$$
\frac{\partial f}{\partial t}+\mathbf{v} \cdot \nabla_{\mathbf{x}} f+\varepsilon_{R}\left(-\nabla \Phi+\mathbf{v} \times\left(\nabla \times\left(0,0, A_{z}\right)\right) \cdot \nabla_{\mathbf{v}} f=0\right.
$$




$$
\begin{aligned}
\epsilon^{2} \frac{\partial^{2} \Phi}{\partial t^{2}}-\nabla^{2} \Phi & =\rho / \varepsilon_{R}, \quad \rho(\mathbf{x}, t)=\int_{\mathbf{v}} f(\mathbf{x}, \mathbf{v}, t) d \mathbf{v} \\
\epsilon^{2} \frac{\partial^{2} A_{z}}{\partial t^{2}}-\nabla^{2} A_{z} & =\mu_{R} J_{z}, \quad J_{z}(\mathbf{x}, t)=\int_{\mathbf{v}} v_{z} f(\mathbf{x}, \mathbf{v}, t) d \mathbf{v}
\end{aligned}
$$

where $\epsilon=\frac{L}{c T}=\frac{V}{c}$, as in the electrostatic case. For the Bennett pinch problem in Section 5.2 , we choose $\varepsilon_{R}=1$.

\section{Appendix B. Von Neumann Analysis}

Appendix B.1. Semi-Discrete Von Neumann Analysis

In this section, we provide semi-discrete Von Neumann stability analyses and dispersion relations for the semi-discrete schemes derived in Section 3. These build on similar analyses for related, but different, schemes given in [4].

Appendix B.1.1. Diffusive Scheme

Substituting the ansatz $u^{n}=e^{i(k \cdot x-\tilde{\omega} n \Delta t)}$ into

$$
\left(-\frac{1}{\alpha_{\sqrt{2}}^{2}} \nabla^{2}+1\right) u^{n+1}=\frac{1}{2}\left(5 u^{n}-4 u^{n-1}+u^{n-2}\right),
$$

we obtain a polynomial

$$
\lambda^{3}-4 \lambda^{2}+5 \lambda-2\left(1+z^{2}\right)=0
$$

where $\lambda=e^{i \tilde{\omega} \Delta t}, z=|k| / \alpha_{\sqrt{2}}^{2}$. The three roots tell us about possible modes $u^{n}=e^{i k \cdot x} \lambda^{-n}$.

A necessary condition for stability is $\lambda \geq 1$ for all roots.

The first root,

$$
\begin{aligned}
\lambda_{1}= & \frac{4}{3}+\frac{1}{3}\left(27 z^{2}+3 \sqrt{3} \sqrt{27 z^{4}+2 z^{2}}+1\right)^{1 / 3}+ \\
& \frac{1}{3\left(27 z^{2}+3 \sqrt{3} \sqrt{27 z^{4}+2 z^{2}}+1\right)^{1 / 3}}
\end{aligned}
$$

corresponds to a spurious nonpropagating mode of the form $u^{n}=e^{i k \cdot x} \lambda_{1}^{-n}$. Since $\lambda_{1} \geq 2$ for all $z=|k| c \Delta t / \sqrt{2} \geq 0$, the mode rapidly decays and poses no threat to stability.

The other two roots are a pair of complex conjugates:

$$
\begin{aligned}
\lambda_{2 / 3}= & -\frac{1}{6}(1 \mp i \sqrt{3})\left(27 z^{2}+3 \sqrt{3} \sqrt{27 z^{4}+2 z^{2}}+1\right)^{1 / 3}- \\
& -\frac{1 \pm i \sqrt{3}}{6\left(27 z^{2}+3 \sqrt{3} \sqrt{27 z^{4}+2 z^{2}}+1\right)^{1 / 3}}+4 / 3 \\
\approx & 1 \pm i \sqrt{2} z-z^{2} \mp i \frac{5 \sqrt{2}}{4} z^{3}+4 z^{4} \pm i \frac{231 \sqrt{2}}{32} z^{5}+O\left(z^{6}\right) \text { as } z \rightarrow 0 .
\end{aligned}
$$


We can show that

$$
\left|\lambda_{2 / 3}\right|^{2}=\frac{16}{9}+\frac{4 W^{4}-16 W^{3}-4 W^{2}-16 W+4}{36 W^{2}} \geq 1 \quad \text { for all } W \geq 1
$$

where $W=\left(27 z^{2}+3 \sqrt{3} \sqrt{27 z^{4}+2 z^{2}}+1\right)^{1 / 3} \geq 1$ for all $z \geq 0$.

Since $\lambda=e^{i \tilde{\omega} \Delta t}$, we have

$$
\begin{aligned}
\tilde{\omega} & =\frac{1}{i \Delta t} \log (\lambda) \\
& \approx \frac{1}{i \Delta t} \log \left(1+i \sqrt{2} z-z^{2}-i \frac{5 \sqrt{2}}{4} z^{3}+4 z^{4}+i \frac{231 \sqrt{2}}{32} z^{5}+O\left(z^{6}\right)\right) \\
& \approx|k| c\left(1-\frac{11(|k| c \Delta t)^{2}}{24}-i \frac{(|k| c \Delta t)^{3}}{2}-\frac{15(|k| c \Delta t)^{4}}{16}+O\left((|k| c \Delta t)^{5}\right)\right)
\end{aligned}
$$

So the phase error is $\left|\frac{\tilde{\omega}}{|k| c}-1\right|=O\left((|k| c \Delta t)^{2}\right)$.

The presence of the imaginary third term in the expansion shows that $u^{n} \sim e^{\left.-i n\left(-i(|k| c \Delta t)^{3}\right) \Delta t\right)}=$ $e^{-n(|k| c)^{3} \Delta t^{4} / 2}$, causing the mode to decay. This is why we term this scheme diffusive (or dissipative).

\section{Appendix B.1.2. Dispersive Scheme}

Substitute ansatz $u^{n}=e^{i(k \cdot x-\tilde{\omega} n \Delta t)}$ into

$$
\left(-\frac{1}{\alpha_{2}^{2}} \nabla^{2}+1\right)\left(u^{n+1}+2 u^{n}+u^{n-1}\right)=4 u^{n}
$$

Obtain a polynomial

$$
\lambda^{2}+2\left(\frac{|k|^{2}-\alpha_{2}^{2}}{|k|^{2}+\alpha_{2}^{2}}\right) \lambda+1=0
$$

where $\lambda=e^{i \tilde{\omega} \Delta t}$.

We can solve to obtain $\lambda_{1 / 2}=\frac{\alpha_{2} \pm i|k|}{\alpha_{2} \mp i|k|}$, which gives $\left|\lambda_{1 / 2}\right|=1$, meaning this scheme is non-dissipative.

Noting that $\cos (\tilde{\omega} \Delta t)=\frac{1}{2}\left(\lambda_{1}+\lambda_{2}\right)$, and defining $z=|k| / \alpha_{2}=|k| c \Delta t / 2$, we obtain

$$
\begin{aligned}
\tilde{\omega} & =\frac{2}{\Delta t} \arccos \left(\sqrt{\frac{\alpha_{2}^{2}}{|k|^{2}+\alpha_{2}^{2}}}\right)=\frac{2}{\Delta t} \arccos \left(\sqrt{\frac{1}{1+z^{2}}}\right) \\
& \approx \frac{2}{\Delta t}\left(z-z^{3} / 3+O\left(z^{5}\right)\right) \\
& \approx|k| c\left(1-\frac{1}{12}(|k| c \Delta t)^{2}+O\left((|k| c \Delta t)^{4}\right)\right)
\end{aligned}
$$

So the phase error is $\left|\frac{\tilde{\omega}}{|k| c}-1\right|=O\left((|k| c \Delta t)^{2}\right)$. Moreover, $\tilde{\omega}$ is real, owing to the non-dissipative nature of the scheme. 


\section{Appendix B.2. Fully Discrete Von Neumann Analysis}

In this section, we provide fully discrete Von Neuman analyses for the two fully discrete schemes derived in Section 3, and show that they are unconditionally stable, in the sense of Von Neumann analysis. Combining the quadrature rules and exponential recursion, and ignoring boundaries, we can write

$$
\begin{aligned}
I[f]\left(x_{j}\right) & =\frac{\alpha}{2} \int_{-\infty}^{\infty} f\left(x^{\prime}\right) e^{-\alpha\left|x_{j}-x^{\prime}\right|} d x^{\prime} \approx \\
& \approx I_{h}\left[f_{j}\right]=P f_{j}+\frac{1}{2} Q\left(f_{j+1}+f_{j-1}\right)+R\left(f_{j+1}-2 f_{j}+f_{j-1}\right)+ \\
& +\frac{1}{2} \sum_{k=1}^{\infty} e^{-\nu k}\left[P\left(f_{j+k}+f_{j-k}\right)+Q\left(f_{j+k+1}+f_{j-k-1}\right)+\right. \\
& \left.+R\left(f_{j+k+1}-2 f_{j+k}+f_{j+k-1}+f_{j-k+1}-2 f_{j-k}+f_{j-k-1}\right)\right]
\end{aligned}
$$

with $\nu=\alpha h=\alpha \Delta x$ and $P, Q$, and $R$ defined as in Section 3 (For ease of presentation we write $\alpha$ instead of $\alpha_{\beta}$ for both diffusive and dispersive schemes, which does not alter the analysis.)

Using the discrete convolution operator $I_{h}$, and ignoring sources and boundaries, we can write the diffusive version of the fully discrete scheme as

$$
u_{j}^{n+1}=\frac{1}{2} I_{h}\left[5 u_{j}^{n}-4 u_{j}^{n-1}+u_{j}^{n-2}\right]
$$

and the dispersive scheme as

$$
u_{j}^{n+1}+2 u_{j}^{n}+u_{j}^{n-1}=4 I_{h}\left[u_{j}^{n}\right]
$$

Defining $I_{h, x}$ and $I_{h, y}$ as the discrete convolution operators acting in the $x$ - and $y$ directions, and again ignoring sources and boundaries, we can write the diffusive version of the 2D fully discrete scheme as

$$
u_{j k}^{n+1}=\frac{1}{2} I_{h, x}\left[I_{h, y}\left[5 u_{j k}^{n}-4 u_{j k}^{n-1}+u_{j k}^{n-2}\right]\right]
$$

and the 2D fully dispersive scheme as

$$
u_{j k}^{n+1}+2 u_{j k}^{n}+u_{j k}^{n-1}=4 I_{h, x}\left[I_{h, y}\left[u_{j k}^{n}\right]\right]
$$

We will refer to these as free-space schemes. We now state a stability theorem based on the Von Neumann analysis of the schemes.

Theorem. The fully discrete dispersive and diffusive free-space schemes in one dimension are unconditionally stable. In higher dimensions, the corresponding dimensionally-split schemes are also unconditionally stable.

To prove the stability theorem, we consider some properties of the discrete covolution operator. 
Lemma. Define the amplification factor $A(\tilde{k}, \nu)=e^{-i \tilde{k} j \Delta x} I_{h}\left[e^{i \tilde{k} j \Delta x}\right]$. Then $A(\tilde{k}, \nu)$ is welldefined (independent of $j$ ), and the following hold.

- If $\nu>0$ and $0<|\tilde{k} \Delta x| \leq \pi$, then $0<A(\tilde{k}, \nu)<1$.

- If $\nu>0$, then $A(0, \nu)=1$.

- For any $0<|\tilde{k} \Delta x| \leq \pi, \lim _{\nu \rightarrow 0^{+}} A(\tilde{k}, \nu)=0$.

- For any $0<|\tilde{k} \Delta x| \leq \pi, \lim _{\nu \rightarrow \infty} A(\tilde{k}, \nu)=1$.

Proof of the Lemma. We calculate:

$$
\begin{aligned}
& A(\tilde{k}, \nu)= e^{-i \tilde{k} j \Delta x} I_{h}\left[e^{i \tilde{k} j \Delta x}\right] \\
&= P\left(1+\sum_{k=1}^{\infty} e^{-k \nu} \cos (k \tilde{k} \Delta x)\right)+ \\
&+Q\left(e^{\nu} \sum_{k=1}^{\infty} e^{-k \nu} \cos (k \tilde{k} \Delta x)\right)+ \\
&+2 R(\cos (\tilde{k} \Delta x)-1)\left(1+\sum_{k=1}^{\infty} e^{-k \nu} \cos (k \tilde{k} \Delta x)\right) \\
&= 1+T, \\
& T=\frac{\frac{d^{2}+d}{\nu}(\cos (\tilde{k} \Delta x)-1)^{2}-2 \frac{1-d}{\nu^{2}}(d \cos (\tilde{k} \Delta x)-1)(\cos (\tilde{k} \Delta x)-1)}{d^{2}-2 \cos (\tilde{k} \Delta x) d+1}
\end{aligned}
$$

with $d=e^{-\nu}$.

If $\tilde{k} \Delta x=0$ and $\nu>0$, then $T=0$. If $0<|\tilde{k} \Delta x| \leq \pi$, some calculus shows $-1<T<0$ for any $\nu>0$, and $\lim _{\nu \rightarrow 0^{+}} T=-1$, and $\lim _{\nu \rightarrow \infty} T=0$.

Proof of the Theorem. We first prove that each one-dimensional free-space fully discrete scheme is unconditionally stable, then describe the extension of the result to multiple dimensions for the dimensionally-split schemes.

\section{Diffusive Scheme}

Consider applying the fully discrete diffusive scheme (B.4) to $u_{j}^{n}=e^{i(\tilde{k} j \Delta x-\tilde{\omega} n \Delta t)}$. We obtain a polynomial

$$
\lambda^{3}-4 \lambda^{2}+5 \lambda-z=0
$$

where $\lambda=e^{i \tilde{\omega} \Delta t}$ and $z=2 / A(\tilde{k}, \nu)$. Note that the lemma implies $z \geq 2$ for all $\tilde{k}$ and $\Delta x, \Delta t>0$. The condition for stability is $|\lambda| \geq 1$ for all roots of the polynomial, which we verify below. 
The first root corresponds to a spurious mode:

$$
\begin{aligned}
\lambda_{1}= & \frac{4}{3}+\frac{\sqrt[3]{3 \sqrt{3} \sqrt{27 z^{2}-104 z+100}+27 z-52}}{3 \sqrt[3]{2}}+ \\
& +\frac{\sqrt[3]{2}}{3 \sqrt[3]{3 \sqrt{3} \sqrt{27 z^{2}-104 z+100}+27 z-52}}
\end{aligned}
$$

We can show that

$$
\lambda_{1}=\frac{W^{2}+4 W+1}{3 W} \geq 2 \text { for all } W \geq 1
$$

where $W=\frac{\sqrt[3]{3 \sqrt{3} \sqrt{27 z^{2}-104 z+100}+27 z-52}}{\sqrt[3]{2}} \geq 1$ for $z \geq 2$.

The other roots are pair of complex conjugates:

$$
\begin{aligned}
\lambda_{2 / 3}= & \frac{4}{3}-(1 \mp i \sqrt{3}) \frac{\sqrt[3]{3 \sqrt{3} \sqrt{27 z^{2}-104 z+100}+27 z-52}}{6 \sqrt[3]{2}}- \\
& -(1 \pm i \sqrt{3}) \frac{\sqrt[3]{2}}{6 \sqrt[3]{3 \sqrt{3} \sqrt{27 z^{2}-104 z+100}+27 z-52}}
\end{aligned}
$$

We can show that

$$
\left|\lambda_{2 / 3}\right|^{2}=\frac{4 W^{4}-16 W^{3}+60 W^{2}-16 W+4}{36 W^{2}} \geq 1 \text { for all } W \geq 1
$$

where $W=\frac{\sqrt[3]{3 \sqrt{3} \sqrt{27 z^{2}-104 z+100}+27 z-52}}{\sqrt[3]{2}} \geq 1$ for $z \geq 2$.

This proves the theorem in the case of the one dimensional diffusive scheme.

\section{Dispersive Scheme}

In this section, we prove that the one-dimensional free-space fully discrete dispersive scheme is unconditionally stable and non-dissipative. Consider applying the fully discrete dispersive scheme (B.5) to $u_{j}^{n}=e^{i(\tilde{k} j \Delta x-\tilde{\omega} n \Delta t)}$. We obtain a polynomial

$$
\lambda^{2}+(2-4 z) \lambda+1=0
$$

where $\lambda=e^{i \tilde{\omega} \Delta t}$ and $z=A(\tilde{k}, \nu)$. Note that the lemma implies $0 \leq z \leq 1$ for all $\tilde{k}$ and $\Delta x, \Delta t>0$. We can solve to obtain the roots $\lambda_{1 / 2}=(2 z-1) \pm i \sqrt{(1-z) z}$. We can show $\left|\lambda_{1 / 2}\right|=1$ for all $0 \leq z \leq 1$, so the fully discrete dispersive scheme is unconditionally stable, and non-dissipative. This proves the theorem in the case of the one dimensional dispersive scheme.

\section{Extension to Higher Dimensions}

When applying the dimensionally-split two-dimensional schemes (B.6), (B.7) to $u_{j k}^{n}=$ $e^{i\left(\tilde{k}_{x} j \Delta x+\tilde{k}_{y} k \Delta y-\tilde{\omega} n \Delta t\right)}$, we obtain the same Von Neumann polynomials as in the $1 \mathrm{D}$ case, 
and basically the same stability analysis can be repeated. This can be easily extended to dimensionally-split schemes in any dimension.

This Von Neumann analysis does not take into consideration the effects of boundary conditions, and in principle, certain numerical boundary conditions could result in instability. In the test problems presented in $[4,5,6]$ as well as the present work, the stability of the method seems robust under a variety of numerical boundary conditions, though in [6], it was found necessary to include artificial dissipation to stabilize an embedded boundary method for Neumann boundary conditions. A stability analysis for some 1D fully discrete schemes (slightly different from those presented here) with numerical Dirichlet and Neumann boundary conditions was carried out in [4], showing unconditional stability in those schemes. A similar stability analysis could also be carried out for the schemes considered in this work to study the stability of these schemes under the inclusion of numerical boundary conditions.

\section{Appendix C. Details of the Fast Particle Convolution Algorithm}

\section{Appendix C.1. Fast Convolution Algorithm in 1D}

We now describe the algorithm used for the fast exact evaluation of the convolution of charge and current density source terms due to particles. It has two main steps. There is a local deposit step and then a recursive sweep step. This basic structure is the same in all dimensions. For definiteness, we describe the application of the algorithm to linear particle shapes in one and two dimensions. However, it may be generalized to any separable particle shapes with compact support in any dimension. Note that this includes many widely used particle shapes in PIC algorithms, namely typical spline-based particle shapes and (suitably cut-off) Gaussian particle shapes. For the case of the charge density integral, the particle shape function $S_{p}$ below is replaced by $q_{p} S_{p}$ and its contribution summed to the charge density integral, and for the case of the current density integral, $S_{p}$ is replaced by $\mathbf{v}_{p} q_{p} S_{p}$ and its contribution summed to the current density integral.

\section{Appendix C.1.1. Local Deposit Step in 1D}

Consider particle $p$ located in the cell $\left[x_{m}, x_{m+1}\right]$ in a uniform grid with cell length $\Delta x$. Let $S_{p}(x)$ be the shape function of the particle. Assume that the support of $S_{p}$ has length $2 r \Delta x$ for some integer $r$. The local deposit step then consists of analytically evaluating the integrals

$$
\begin{aligned}
J_{j+1}^{L, p} & =\frac{\alpha}{2} \int_{x_{j}}^{x_{j+1}} S_{p}\left(x^{\prime}\right) e^{-\alpha\left(x_{j+1}-x^{\prime}\right)} d x^{\prime} \\
J_{j}^{R, p} & =\frac{\alpha}{2} \int_{x_{j}}^{x_{j+1}} S_{p}\left(x^{\prime}\right) e^{-\alpha\left(x^{\prime}-x_{j}\right)} d x^{\prime}
\end{aligned}
$$

for $j=m-r, \ldots, m, \ldots, m+r$ and for each particle $p$, and summing their values on the grid. 
For linear particle shapes (corresponding to $r=1$ ), we have $S_{p}(x)=S\left(\frac{x-x_{p}}{\Delta x}\right)$, where

$$
S(z)= \begin{cases}1-|z| & |z|<1 \\ 0 & |z| \geq 1\end{cases}
$$

Let $a=\left(x_{p}-x_{m}\right) / \Delta x$, where $x_{p}$ is the location of the particle. We define $\nu=\alpha \Delta x$ and perform the change of variables $z=\left(x-x_{p}\right) / \Delta x$ in the convolution integral. For linear particle shapes, we then have the situation displayed in Figure C.10.

The desired integrals are then easily evaluated for linear particle shapes:

$$
\begin{aligned}
J_{m}^{L, p} & =\frac{\nu}{2} \int_{-1}^{-a}\left(1+z^{\prime}\right) e^{-\nu\left(-a-z^{\prime}\right)} d z^{\prime} \\
& =\left(((1-a) \nu-1)+e^{\nu a} e^{-\nu}\right) / 2 \nu \\
J_{m+1}^{L, p} & =\frac{\nu}{2} \int_{-a}^{1-a}\left(1-\left|z^{\prime}\right|\right) e^{-\nu\left(1-a-z^{\prime}\right)} d z^{\prime} \\
& =\left(e^{-\nu}((a-1) \nu+1)+a \nu+1-2 e^{-\nu} e^{\nu a}\right) / 2 \nu \\
J_{m+2}^{L, p} & =\frac{\nu}{2} \int_{1-a}^{1}\left(1-z^{\prime}\right) e^{-\nu\left(2-a-z^{\prime}\right)} d z^{\prime} \\
& =\left(e^{-\nu}(-a \nu-1)+e^{-\nu} e^{\nu a}\right) / 2 \nu \\
J_{m-1}^{R, p} & =\frac{\nu}{2} \int_{-1}^{-a}\left(1+z^{\prime}\right) e^{-\nu\left(z^{\prime}-(-1-a)\right)} d z^{\prime} \\
& =\left(e^{-\nu}((a-1) \nu-1)+e^{-\nu a}\right) / 2 \nu \\
J_{m}^{R, p} & =\frac{\nu}{2} \int_{-a}^{1-a}\left(1-\left|z^{\prime}\right|\right) e^{-\nu\left(z^{\prime}-(-a)\right)} d z^{\prime} \\
& =\left((1-a) \nu+1+e^{-\nu}(-a \nu+1)-2 e^{-\nu a}\right) / 2 \nu \\
J_{m+1}^{R, p} & =\frac{\nu}{2} \int_{1-a}^{1}\left(1-z^{\prime}\right) e^{-\nu\left(z^{\prime}-(1-a)\right)} d z^{\prime} \\
= & \left(a \nu-1+e^{-\nu a}\right) / 2 \nu
\end{aligned}
$$

Note that just one evaluation of an exponential function is required per particle (namely $\left.e^{\nu a}\right)$. To obtain the total local deposit, we simply sum the particle contributions on to the grid. Let $N_{p}$ be the total number of particles. The algorithm for the local deposit step is given by:

Initialize $J_{k}^{L}=J_{k}^{R}=0$ for all $k$.

for $p=1: N_{p}$ do

Particle $p$ located in cell $\left[x_{m}, x_{m+1}\right]$.

for $j=m-r: m+r$ do

Compute $J_{j+1}^{L, p}, J_{j}^{R, p}$

Deposit $J_{j+1}^{L}=J_{j+1}^{L}+J_{j+1}^{L, p}, J_{j}^{R}=J_{j}^{R}+J_{j}^{R, p}$ 


\section{end for \\ end for}

Note that the local deposit step costs $O\left(N_{p}\right)$ operations.

Appendix C.1.2. Recursive Sweep Step in 1D

Once we have performed the local deposit step, we complete the evaluation of the particle integral with a recursive sweep step. Suppose we have $N$ gridpoints, $x_{1}, \ldots, x_{N}$. The algorithm for the recursive sweep step is given by:

Initialize $I_{1}^{L}=I_{N}^{R}=0$

for $j=1: N-1$ do

$$
\begin{aligned}
& I_{j+1}^{L}=J_{j+1}^{L}+e^{-\nu} I_{j}^{L} \\
& I_{N-j}^{R}=J_{N-j}^{R}+e^{-\nu} I_{N-j+1}^{R}
\end{aligned}
$$

\section{end for}

$I=I^{L}+I^{R}$

Note that the recursive sweep step costs $O(N)$ operations.

\section{Appendix C.2. Fast Convolution Algorithm in 2D}

For a separable particle shape $S(x, y)=S_{x}(x) S_{y}(y)$, we have

$$
\begin{aligned}
I_{x}\left[I_{y}[S]\right](x, y)= & I_{x}\left[S_{x}\right](x) \cdot I_{y}\left[S_{y}\right](y) \\
= & \left(I_{x}^{L}\left[S_{x}\right](x)+I_{x}^{R}\left[S_{x}\right](x)\right) \cdot\left(I_{y}^{D}\left[S_{y}\right](y)+I_{y}^{U}\left[S_{y}\right](y)\right) \\
= & I_{x}^{L}\left[S_{x}\right](x) \cdot I_{y}^{D}\left[S_{y}\right](y)+I_{x}^{L}\left[S_{x}\right](x) \cdot I_{y}^{U}\left[S_{y}\right](y)+ \\
& +I_{x}^{R}\left[S_{x}\right](x) \cdot I_{y}^{D}\left[S_{y}\right](y)+I_{x}^{R}\left[S_{x}\right](x) \cdot I_{y}^{U}\left[S_{y}\right](y)
\end{aligned}
$$

This is suggestive of how we will build the $2 \mathrm{D}$ algorithm.

\section{Appendix C.2.1. Local Deposit Step in 2D}

Consider particle $p$ centered at $\left(x_{p}, y_{p}\right) \in\left[x_{m}, x_{m+1}\right] \times\left[y_{n}, y_{n+1}\right]$, with separable particle shape $S_{p}(x, y)=S\left(\frac{x-x_{p}}{\Delta x}\right) S\left(\frac{y-y_{p}}{\Delta y}\right)$ with linear particle shape $S$ defined as above. The support of the particle shape is shown in Figure C.11.

In the local deposit step, we form a tensor product on the grid as suggested by the above decomposition. Note that a total of 12 local integrals must be evaluated for each particle, then summed onto the grid as a tensor product.

Initialize $J_{j, k}^{L U}=J_{j, k}^{L D}=J_{j, k}^{R U}=J_{j, k}^{R D}=0$ for all $j, k$.

for $p=1: N_{p}$ do

Particle $p$ located in cell $\left[x_{m}, x_{m+1}\right] \times\left[y_{n}, y_{n+1}\right]$.

for $j=m-r: m+r, k=n-r: n+r$ do

Compute $J_{j+1}^{L, p}, J_{j}^{R, p}, J_{k+1}^{D, p}, J_{k}^{U, p}$

Deposit:

$J_{j+1, k+1}^{L D}=J_{j+1, k+1}^{L D}+J_{j+1}^{L, p} \cdot J_{k+1}^{D, p}$

$J_{j+1, k}^{L U}=J_{j+1, k}^{L U}+J_{j+1}^{L, p} \cdot J_{k}^{U, p}$

$J_{j, k+1}^{R D}=J_{j, k+1}^{R D}+J_{j}^{R, p} \cdot J_{k+1}^{D, p}$ 


$$
\begin{aligned}
& \qquad J_{j, k}^{R U}=J_{j, k}^{R U}+J_{j}^{R, p} \cdot J_{k}^{U, p} \\
& \text { end for } \\
& \text { end for }
\end{aligned}
$$

Appendix C.2.2. Recursive Sweep Step in 2D

The recursive sweep step is similar to the $1 \mathrm{D}$ case, and is given below.

$$
\begin{aligned}
& \text { for } k=1: N_{y} \text { do } \\
& \quad \text { Initialize } I_{1, k}^{L D}=I_{1, k}^{L U}=I_{N_{x}, k}^{R D}=I_{N_{x}, k}^{R U}=0 \\
& \quad \text { for } j=1: N_{x}-1 \text { do } \\
& \quad I_{j+1, k}^{L D}=J_{j+1, k}^{L D}+e^{-\nu} I_{j, k}^{L D} \\
& \quad I_{j+1, k}^{L U}=J_{j+1, k}^{L U}+e^{-\nu} I_{j, k}^{L U} \\
& \quad I_{N_{x}-j, k}^{R D}=J_{N_{x}-j, k}^{R D}+e^{-\nu} I_{N_{x}-j+1, k}^{R D} \\
& \quad I_{N_{x}-j, k}^{R U}=J_{N_{x}-j, k}^{R U}+e^{-\nu} I_{N_{x}-j+1, k}^{R U} \\
& \quad \text { end for } \\
& \text { end for } \\
& J^{D}=I^{L D}+I^{R D} \\
& J^{U}=I^{L U}+I^{R U} \\
& \text { for } j=1: N_{x} \text { do } \\
& \quad \text { Initialize } I_{j, 1}^{D}=I_{j, N_{y}}^{U}=0 . \\
& \quad \text { for } k=1: N_{y}-1 \text { do } \\
& \quad I_{j, k+1}^{D}=J_{j, k+1}^{D}+e^{-\nu} I_{j, k}^{D} \\
& \quad I_{j, N_{y}-k}^{U}=J_{j, N_{y}-k}^{U}+e^{-\nu} I_{j, N_{y}-k+1}^{U} \\
& \quad \text { end for } \\
& \text { end for } \\
& I=I^{D}+I^{U}
\end{aligned}
$$

\section{end for}

Both 1D and 2D overall algorithms cost $O\left(N_{p}+N\right)$ operations, where $N$ is the total number of gridpoints. Since in a typical PIC simulation, $N_{p}>N$, the cost of the overall algorithm is dominated by the local deposit step.

\section{Appendix D. A Time-Staggered Scheme}

In this work, we chose to use the diffusive version of the implicit wave solver, as its slight numerical dissipation provided a means of reducing noise in our simulations. However, this diffusive solver has some disadvantages compared to the dispersive scheme. First, it contains a source term at time level $n+1$, which complicates the implementation of second- and higherorder accurate particle push methods in electromagnetic problems. Second, its truncation error is larger than that of the dispersive scheme, a fact demonstrated by the leading order phase error terms in (B.1) and (B.2). Third, unlike the dispersive scheme which can naturally be extended to higher-order accuracy with a Lax-Wendroff approach, the diffusive scheme is not easily extended to higher-order accuracy, as using a higher-order BDF formula will not necessarily result in an unconditionally stable scheme [5]. Finally, the numerical dissipation, while useful in controlling noise in simulations, is of a fixed strength (for a given $\Delta t$ ) and not tunable, whereas an tunable artificial dissipation method was developed for the dispersive 
scheme [6]. Hence, future work will be focused on the development of the dispersive scheme and its generalizations. We will consider the use of higher-order particle shape functions (for which our fast particle integration algorithm is easily adapted) and the tunable artificial dissipation method of [6] as means of reducing noise. In order to obtain higher-order accurate solutions to Maxwell's equations, we may apply higher-order methods presented in [5].

We now propose a second-order accurate, time-staggered Maxwell solver and PIC method based on the dispersive wave solver and a staggered grid in time and space, using the explicit leapfrog algorithm for the particles. This method seems to be our most natural analogue of the classical FDTD-Leapfrog EM-PIC method.

The scalar potential $\Phi$ and particle positions $\mathbf{x}_{p, i}$ (and charge density $\rho$ ) are collocated at integer time steps, while the vector potential $\mathbf{A}$ and particle positions $\mathbf{v}_{p, i}$ (and current density $\mathbf{J}$ ) are collocated at half-integer time steps. Given $\mathbf{v}_{p, i}^{n-1 / 2}$ and $\mathbf{x}_{p, i}^{n}$, we have the source terms

$$
\begin{aligned}
\rho^{n}(\mathbf{x}) & =\sum_{i=1}^{N_{p}} q_{i} S\left(\mathbf{x}-\mathbf{x}_{p, i}^{n}\right) \\
\mathbf{J}^{n-1 / 2}(\mathbf{x}) & =\sum_{i=1}^{N_{p}} q_{i} \mathbf{v}_{p, i}^{n-1 / 2}\left(\frac{S\left(\mathbf{x}-\mathbf{x}_{p, i}^{n-1}\right)+S\left(\mathbf{x}-\mathbf{x}_{p, i}^{n}\right)}{2}\right)
\end{aligned}
$$

and we advance the potentials using the dispersive wave solver with the Poisson potential:

$$
\begin{array}{lr}
\mathbf{A}^{n+1 / 2}=\mathbf{F}\left(\mathbf{A}^{n-1 / 2}, \mathbf{A}^{n-3 / 2}, \mathbf{J}^{n-1 / 2}\right), & \text { dispersive wave solver }, \\
-\nabla^{2} \Phi^{n}=\rho^{n} / \epsilon_{0}+\nabla \cdot \frac{\mathbf{A}^{n+1 / 2}-\mathbf{A}^{n-1 / 2}}{\Delta t}, & \text { Poisson potential. }
\end{array}
$$

Having solved for the potentials on an appropriately staggered grid, we calculate the electric and magnetic fields, $\mathbf{E}$ and $\mathbf{B}$ respectively, at time level $n$ by

$$
\begin{aligned}
\mathbf{E}^{n} & =-\nabla \Phi^{n}-\frac{\mathbf{A}^{n+1 / 2}-\mathbf{A}^{n-1 / 2}}{\Delta t} \\
\mathbf{B}^{n} & =\frac{1}{2}\left(\nabla \times \mathbf{A}^{n-1 / 2}+\nabla \times \mathbf{A}^{n+1 / 2}\right)
\end{aligned}
$$

that will satisfy discrete forms of Gauss' law and the divergence-free condition on the magnetic field. (Alternatively, a wave equation may be evolved for $\Phi$ and a hyperbolic divergence cleaning technique may be employed, avoiding the need for a Poisson solve.) These computed fields may then be used to advance the particle positions and velocities as in the classical FDTD-Leapfrog EM-PIC algorithm.

[1] Charles Kennedy Birdsall and Allan Bruce Langdon. Plasma Physics Via Computer Simulaition. CRC Press, 2005.

[2] Roger W Hockney and James W Eastwood. Computer simulation using particles. CRC Press, 1988. 
[3] John P Verboncoeur. Particle simulation of plasmas: review and advances. Plasma Physics and Controlled Fusion, 47(5A):A231, 2005.

[4] Matthew Causley, Andrew Christlieb, Benjamin Ong, and Lee Van Groningen. Method of lines transpose: An implicit solution to the wave equation. Mathematics of Computation, 2014.

[5] Matthew F Causley and Andrew J Christlieb. Higher order a-stable schemes for the wave equation using a successive convolution approach. SIAM Journal on Numerical Analysis, 52(1):220-235, 2014.

[6] M. Causley, A. Christlieb, and E. Wolf. Method of lines transpose: an efficient a-stable solver for wave propagation. Journal of Scientific Computing, (to appear), 2016.

[7] Kane S Yee. Numerical solution of initial boundary value problems involving maxwell's equations. IEEE Trans. Antennas Propag, 14(3):302-307, 1966.

[8] Alen Taflove and Susan C Hagness. Computational electrodynamics: the fdtd method. Artech House Boston, London, 2000.

[9] PJ Mardahl and JP Verboncoeur. Charge conservation in electromagnetic pic codes; spectral comparison of boris/dadi and langdon-marder methods. Computer physics communications, 106(3):219-229, 1997.

[10] John P Verboncoeur. Aliasing of electromagnetic fields in stair step boundaries. Computer physics communications, 164(1):344-352, 2004.

[11] C. S. Meierbachtol, A. D. Greenwood, J. P. Verboncoeur, and B. Shanker. Conformal electromagnetic particle in cell: A review. IEEE Transactions on Plasma Science, 43(11):3778-3793, Nov 2015.

[12] J.-L. Vay, J.-C. Adam, and A. Héron. Asymmetric $\{$ PML $\}$ for the absorption of waves. application to mesh refinement in electromagnetic particle-in-cell plasma simulations. Computer Physics Communications, 164(1-3):171 - 177, 2004. Proceedings of the 18th International Conferene on the Numerical Simulation of Plasmas.

[13] Keizo Fujimoto. A new electromagnetic particle-in-cell model with adaptive mesh refinement for high-performance parallel computation. Journal of Computational Physics, 230(23):8508 - 8526, 2011.

[14] John Villasenor and Oscar Buneman. Rigorous charge conservation for local electromagnetic field solvers. Computer Physics Communications, 69(2):306-316, 1992.

[15] M.E. Innocenti, G. Lapenta, S. Markidis, A. Beck, and A. Vapirev. A multi level multi domain method for particle in cell plasma simulations. Journal of Computational Physics, 238:115 - 140, 2013. 
[16] A. Beck, M.E. Innocenti, G. Lapenta, and S. Markidis. Multi-level multi-domain algorithm implementation for two-dimensional multiscale particle in cell simulations. Journal of Computational Physics, 271:430 - 443, 2014. Frontiers in Computational PhysicsModeling the Earth System.

[17] Rodney J Mason. Implicit moment particle simulation of plasmas. Journal of Computational Physics, 41(2):233 - 244, 1981.

[18] GB Jacobs and Jan S Hesthaven. High-order nodal discontinuous galerkin particle-incell method on unstructured grids. Journal of Computational Physics, 214(1):96-121, 2006.

[19] GB Jacobs and Jan S Hesthaven. Implicit-explicit time integration of a high-order particle-in-cell method with hyperbolic divergence cleaning. Computer Physics Communications, 180(10):1760-1767, 2009.

[20] Fenghua Zheng, Zhizhang Chen, and Jiazong Zhang. A finite-difference time-domain method without the courant stability conditions. Microwave and Guided Wave Letters, IEEE, 9(11):441-443, 1999.

[21] Fenghua Zhen, Zhizhang Chen, and Jiazong Zhang. Toward the development of a threedimensional unconditionally stable finite-difference time-domain method. Microwave Theory and Techniques, IEEE Transactions on, 48(9):1550-1558, 2000.

[22] Takefumi Namiki. 3-d adi-fdtd method-unconditionally stable time-domain algorithm for solving full vector maxwell's equations. Microwave Theory and Techniques, IEEE Transactions on, 48(10):1743-1748, 2000.

[23] Jongwoo Lee and Bengt Fornberg. Some unconditionally stable time stepping methods for the 3d maxwell's equations. Journal of Computational and Applied Mathematics, 166(2):497-523, 2004.

[24] David N Smithe, John R Cary, and Johan A Carlsson. Divergence preservation in the adi algorithms for electromagnetics. Journal of Computational Physics, 228(19):7289-7299, 2009.

[25] Supriyo Dey and Raj Mittra. A locally conformal finite-difference time-domain (fdtd) algorithm for modeling three-dimensional perfectly conducting objects. Microwave and Guided Wave Letters, IEEE, 7(9):273-275, 1997.

[26] Jean-Luc Vay, Irving Haber, and Brendan B. Godfrey. A domain decomposition method for pseudo-spectral electromagnetic simulations of plasmas. Journal of Computational Physics, 243:260 - 268, 2013.

[27] Brendan B. Godfrey, Jean-Luc Vay, and Irving Haber. Numerical stability analysis of the pseudo-spectral analytical time-domain $\{\mathrm{PIC}\}$ algorithm. Journal of Computational Physics, 258:689 - 704, 2014. 
[28] Brendan B. Godfrey and Jean-Luc Vay. Numerical stability of relativistic beam multidimensional $\{\mathrm{PIC}\}$ simulations employing the esirkepov algorithm. Journal of Computational Physics, 248:33 - 46, 2013.

[29] Brendan B. Godfrey and Jean-Luc Vay. Improved numerical cherenkov instability suppression in the generalized $\{\mathrm{PSTD}\}\{\mathrm{PIC}\}$ algorithm. Computer Physics Communications, 196:221 - 225, 2015.

[30] T.Zh. Esirkepov. Exact charge conservation scheme for particle-in-cell simulation with an arbitrary form-factor. Computer Physics Communications, 135(2):144 - 153, 2001.

[31] Andrew D Greenwood, Keith L Cartwright, John W Luginsland, and Ernest A Baca. On the elimination of numerical cerenkov radiation in pic simulations. Journal of Computational Physics, 201(2):665-684, 2004.

[32] Bruce I Cohen, A Bruce Langdon, and Alex Friedman. Implicit time integration for plasma simulation. Journal of Computational Physics, 46(1):15-38, 1982.

[33] JU Brackbill and DW Forslund. An implicit method for electromagnetic plasma simulation in two dimensions. Journal of Computational Physics, 46(2):271-308, 1982.

[34] A Bruce Langdon, Bruce I Cohen, and Alex Friedman. Direct implicit large time-step particle simulation of plasmas. Journal of Computational Physics, 51(1):107-138, 1983.

[35] Dennis W Hewett and A Bruce Langdon. Electromagnetic direct implicit plasma simulation. Journal of Computational Physics, 72(1):121-155, 1987.

[36] Guangye Chen, Luis Chacón, and Daniel C Barnes. An energy- and charge-conserving, implicit, electrostatic particle-in-cell algorithm. Journal of Computational Physics, 230(18):7018-7036, 2011.

[37] Stefano Markidis and Giovanni Lapenta. The energy conserving particle-in-cell method. Journal of Computational Physics, 230(18):7037-7052, 2011.

[38] G. Chen and L. Chacón. A multi-dimensional, energy- and charge-conserving, nonlinearly implicit, electromagnetic vlasov-darwin particle-in-cell algorithm. Computer Physics Communications, 197:73-87, 122015.

[39] Andrew J Christlieb, Robert Krasny, John P Verboncoeur, Jerold W Emhoff, and Iain D Boyd. Grid-free plasma simulation techniques. Plasma Science, IEEE Transactions on, 34(2):149-165, 2006.

[40] Martin Masek and Paul Gibbon. Mesh-free magnetoinductive plasma model. Plasma Science, IEEE Transactions on, 38(9):2377-2382, 2010.

[41] Matthew R Gibbons and Dennis W Hewett. The darwin direct implicit particle-incell (dadipic) method for simulation of low frequency plasma phenomena. Journal of Computational Physics, 120(2):231-247, 1995. 
[42] Francis Filbet and Eric Sonnendrücker. Comparison of eulerian vlasov solvers. Computer Physics Communications, 150(3):247-266, 2003.

[43] E Pohn, Magdi Shoucri, and G Kamelander. Eulerian vlasov codes. Computer physics communications, 166(2):81-93, 2005.

[44] TD Arber and RGL Vann. A critical comparison of eulerian-grid-based vlasov solvers. Journal of computational physics, 180(1):339-357, 2002.

[45] Yingda Cheng, Irene M Gamba, Fengyan Li, and Philip J Morrison. Discontinuous galerkin methods for the vlasov-maxwell equations. SIAM Journal on Numerical Analysis, 52(2):1017-1049, 2014.

[46] Yingda Cheng, Andrew J Christlieb, and Xinghui Zhong. Energy-conserving discontinuous galerkin methods for the vlasov-maxwell system. Journal of Computational Physics, 279:145-173, 2014.

[47] Chio-Zong Cheng and Georg Knorr. The integration of the vlasov equation in configuration space. Journal of Computational Physics, 22(3):330-351, 1976.

[48] Eric Sonnendrücker, Jean Roche, Pierre Bertrand, and Alain Ghizzo. The semilagrangian method for the numerical resolution of the vlasov equation. Journal of computational physics, 149(2):201-220, 1999.

[49] Jing-Mei Qiu and Andrew Christlieb. A conservative high order semi-lagrangian weno method for the vlasov equation. Journal of Computational Physics, 229(4):1130-1149, 2010.

[50] James A Rossmanith and David C Seal. A positivity-preserving high-order semilagrangian discontinuous galerkin scheme for the vlasov-poisson equations. Journal of Computational Physics, 230(16):6203-6232, 2011.

[51] Lev Davidovich Landau and Evgenii Mikhailovich Lifshitï, sï,i. The classical theory of fields, volume 2. Butterworth-Heinemann, 1975.

[52] RL Morse and CW Nielson. Numerical simulation of the weibel instability in one and two dimensions. Physics of Fluids (1958-1988), 14(4):830-840, 1971.

[53] Erich Rothe. Zweidimensionale parabolische randwertaufgaben als grenzfall eindimensionaler randwertaufgaben. Mathematische Annalen, 102(1):650-670, 1930.

[54] Roman Chapko, Rainer Kress, et al. Rothes method for the heat equation and boundary integral equations. Journal of Integral Equations and Applications, 9(1):47-69, 1997.

[55] Mary Catherine A Kropinski and Bryan D Quaife. Fast integral equation methods for rothe's method applied to the isotropic heat equation. Computers $\&$ Mathematics with Applications, 61(9):2436-2446, 2011. 
[56] Mary Catherine A Kropinski and Bryan D Quaife. Fast integral equation methods for the modified helmholtz equation. Journal of Computational Physics, 230(2):425-434, 2011.

[57] Oscar P Bruno and Mark Lyon. High-order unconditionally stable fc-ad solvers for general smooth domains i. basic elements. Journal of Computational Physics, 229(6):2009$2033,2010$.

[58] Mark Lyon and Oscar P Bruno. High-order unconditionally stable fc-ad solvers for general smooth domains ii. elliptic, parabolic and hyperbolic pdes; theoretical considerations. Journal of Computational Physics, 229(9):3358-3381, 2010.

[59] Donald W Peaceman and Henry H Rachford, Jr. The numerical solution of parabolic and elliptic differential equations. Journal of the Society for Industrial $\&$ Applied Mathematics, 3(1):28-41, 1955.

[60] C-D Munz, Pascal Omnes, Rudolf Schneider, Eric Sonnendrücker, and Ursula Voss. Divergence correction techniques for maxwell solvers based on a hyperbolic model. Journal of Computational Physics, 161(2):484-511, 2000.

[61] A Bruce Langdon. On enforcing gauss' law in electromagnetic particle-in-cell codes. Computer Physics Communications, 70(3):447-450, 1992.

[62] H Ralph Lewis, Daniel C Barnes, and Karl J Melendez. The liouville theorem and accurate plasma simulation. Journal of Computational Physics, 69(2):267 - 282, 1987.

[63] RJ Procassini, CK Birdsall, and EC Morse. A fully kinetic, self-consistent particle simulation model of the collisionless plasma-sheath region. Physics of Fluids B: Plasma Physics (1989-1993), 2(12):3191-3205, 1990.

[64] Willard H Bennett. Magnetically self-focussing streams. Physical Review, 45(12):890, 1934.

[65] José A Bittencourt. Fundamentals of plasma physics. Springer, 2004.

[66] J.A Byers, B.I Cohen, W.C Condit, and J.D Hanson. Hybrid simulations of quasineutral phenomena in magnetized plasma. Journal of Computational Physics, 27(3):363 - 396, 1978.

[67] D.W Hewett and C.W Nielson. A multidimensional quasineutral plasma simulation model. Journal of Computational Physics, 29(2):219 - 236, 1978.

[68] Rodney J Mason. An electromagnetic field algorithm for 2d implicit plasma simulation. Journal of Computational Physics, 71(2):429 - 473, 1987.

[69] AS Lipatov. The Hybrid Multiscale Simulation Technology: An Introduction With Application to Astrophysical and Laboratory Plasmas, Monograph. Springer-Verlag, Berlin, Heidelberg, New York, 2002. 
[70] Chet Nieter and John R. Cary. Vorpal: a versatile plasma simulation code. Journal of Computational Physics, 196(2):448 - 473, 2004.

[71] D.R. Welch, D.V. Rose, R.E. Clark, T.C. Genoni, and T.P. Hughes. Implementation of an non-iterative implicit electromagnetic field solver for dense plasma simulation. Computer Physics Communications, 164(1-3):183 - 188, 2004. Proceedings of the 18th International Conferene on the Numerical Simulation of Plasmas.

[72] G.M. Petrov and J. Davis. A two-dimensional electromagnetic field algorithm for highintensity laser-target interactions. Computer Physics Communications, 179(12):868 $880,2008$.

[73] G. M. Petrov and J. Davis. A generalized implicit algorithm for multi-dimensional particle-in-cell simulations in cartesian geometry. Physics of Plasmas, 18(7), 2011.

[74] T. Tuckmantel, A. Pukhov, J. Liljo, and M. Hochbruck. Three-dimensional relativistic particle-in-cell hybrid code based on an exponential integrator. IEEE Transactions on Plasma Science, 38(9):2383-2389, Sept 2010.

[75] T. Tückmantel and A. Pukhov. H-vlpl: A three-dimensional relativistic pic/fluid hybrid code. Journal of Computational Physics, 269:168 - 180, 2014.

[76] Mark J Kushner. Hybrid modelling of low temperature plasmas for fundamental investigations and equipment design. Journal of Physics D: Applied Physics, 42(19):194013, 2009.

[77] V.I. Kolobov and R.R. Arslanbekov. Towards adaptive kinetic-fluid simulations of weakly ionized plasmas. Journal of Computational Physics, 231(3):839 - 869, 2012. Special Issue: Computational Plasma PhysicsSpecial Issue: Computational Plasma Physics.

[78] Masaharu Matsumoto, Yoshihiro Kajimura, Hideyuki Usui, Ikkoh Funaki, and Iku Shinohara. Application of a total variation diminishing scheme to electromagnetic hybrid particle-in-cell plasma simulation. Computer Physics Communications, 183(10):2027 2034, 2012.

[79] Matthew W. Kunz, James M. Stone, and Xue-Ning Bai. Pegasus: A new hybridkinetic particle-in-cell code for astrophysical plasma dynamics. Journal of Computational Physics, 259:154 - 174, 2014.

[80] L. Chacón and G. Chen. A curvilinear, fully implicit, conservative electromagnetic pic algorithm in multiple dimensions. Journal of Computational Physics, 316:578 - 597, 2016.

[81] Lawrence C. Evans. Partial differential equations, volume v. 19 of Graduate studies in mathematics. American Mathematical Society, Providence, R.I., 2nd ed edition, 2010. 
[82] SV Petropavlovsky and SV Tsynkov. Quasi-lacunae of maxwell's equations. SIAM Journal on Applied Mathematics, 71(4):1109-1122, 2011.

[83] Kiyoshi Asano and Seiji Ukai. On the vlasov-poisson limit of the vlasov-maxwell equation. Studies in Mathematics and Its Applications, 18:369-383, 1986.

[84] Jack Schaeffer. The classical limit of the relativistic vlasov-maxwell system. Communications in mathematical physics, 104(3):403-421, 1986. 
Time Step $\mathrm{n}=1$

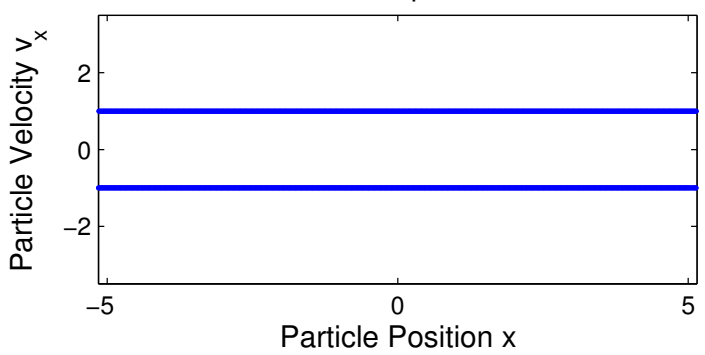

(a)

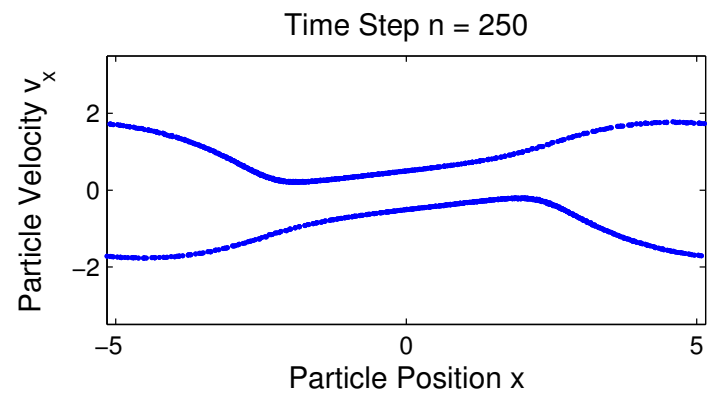

(c)

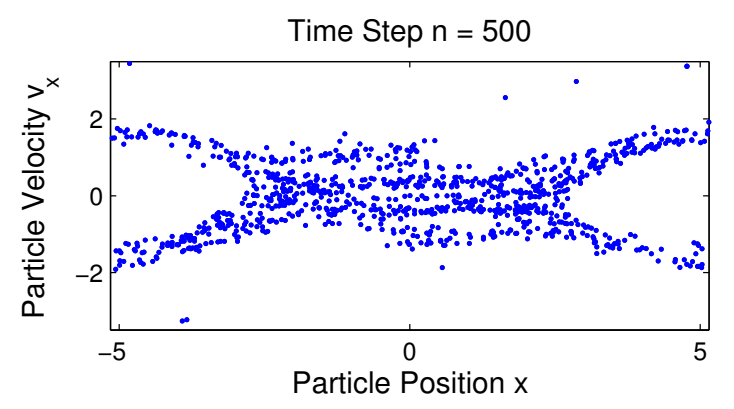

(e)
Time Step $\mathrm{n}=1$

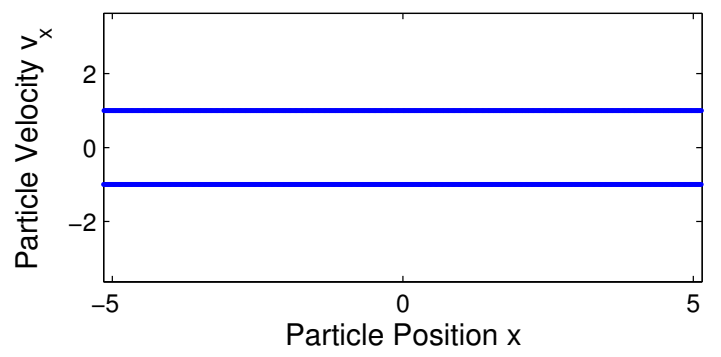

(b)

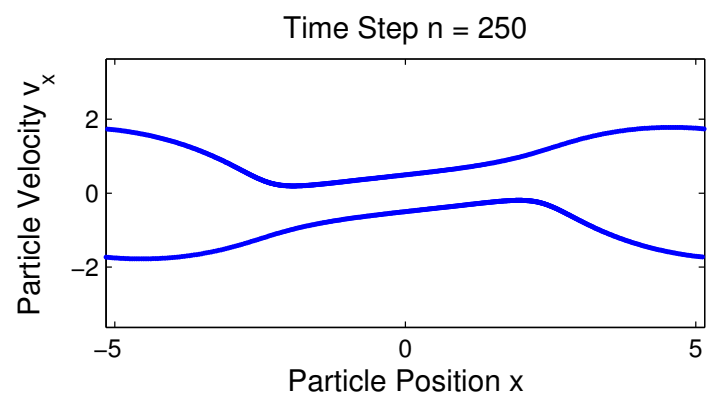

(d)

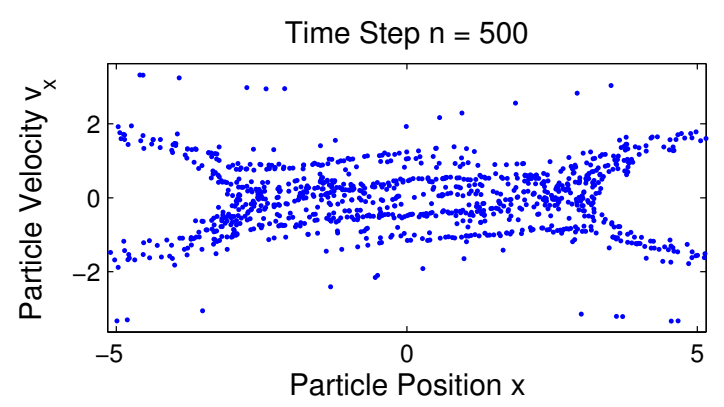

(f)

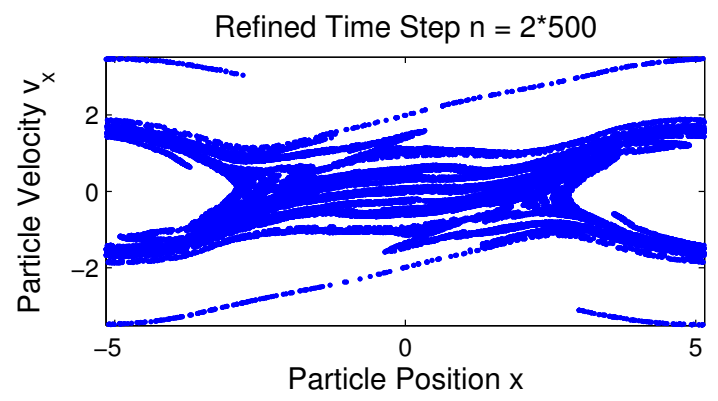

(g)

Figure 3: Selected particle phase space plots for the two stream instability problem. Left column: 1D simulation, right column: 2D simulation, following a fixed slice of particles initialized along the line $y=-L_{y} / 2$, bottom: refined $1 \mathrm{D}$ simulation with grid step size and time step size reduced by a factor of 2 and total number of particles increased by a factor of 400 . 


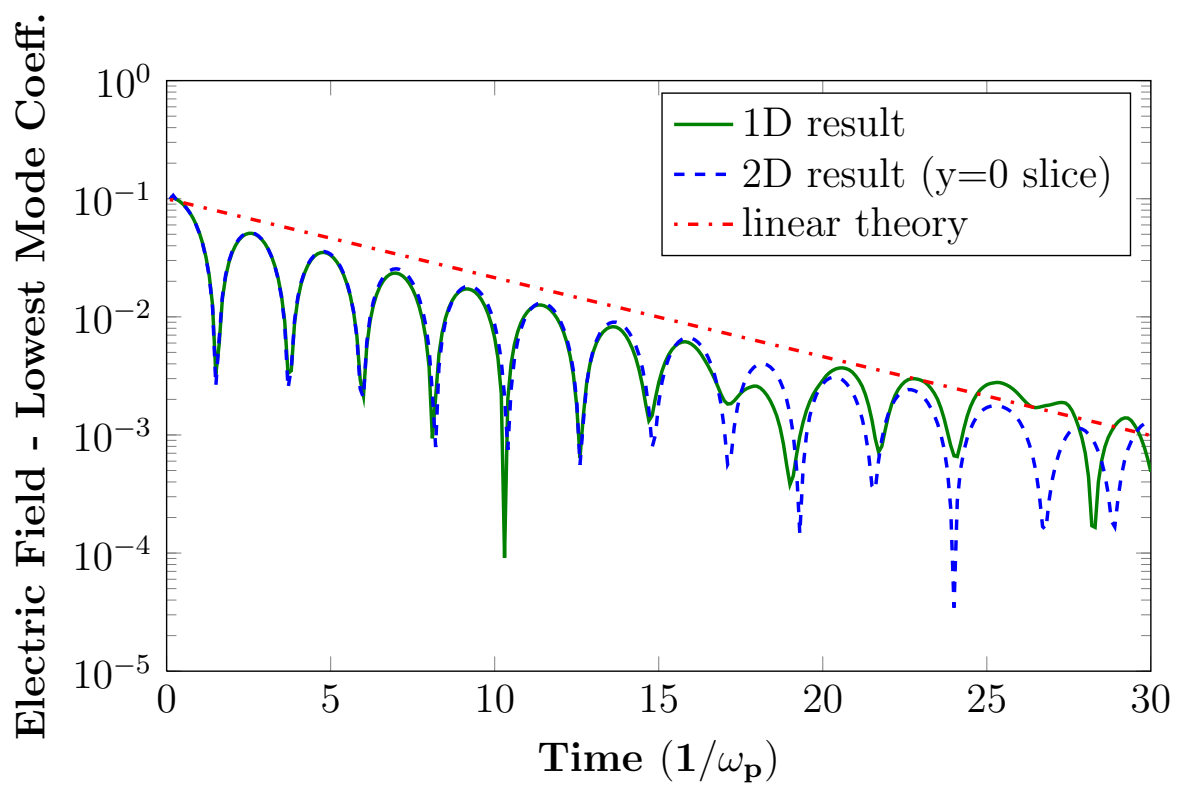

Figure 4: Landau damping of the lowest mode, corresponding to $k=0.5$. Green is the $1 \mathrm{D}$ numerical result, blue is the $2 \mathrm{D}$ numerical result (measured along the central $y=0$ slice), and red is the prediction of linear theory. We see that the correct decay rate is reproduced in our simulations 


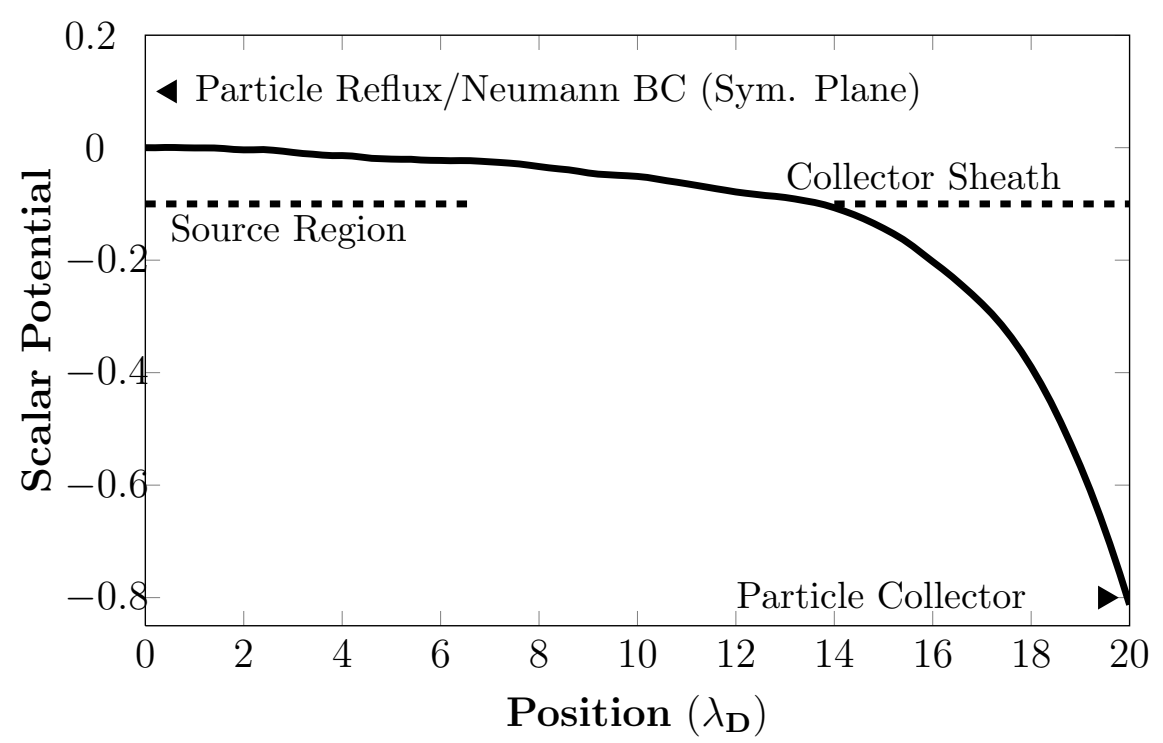

(a)

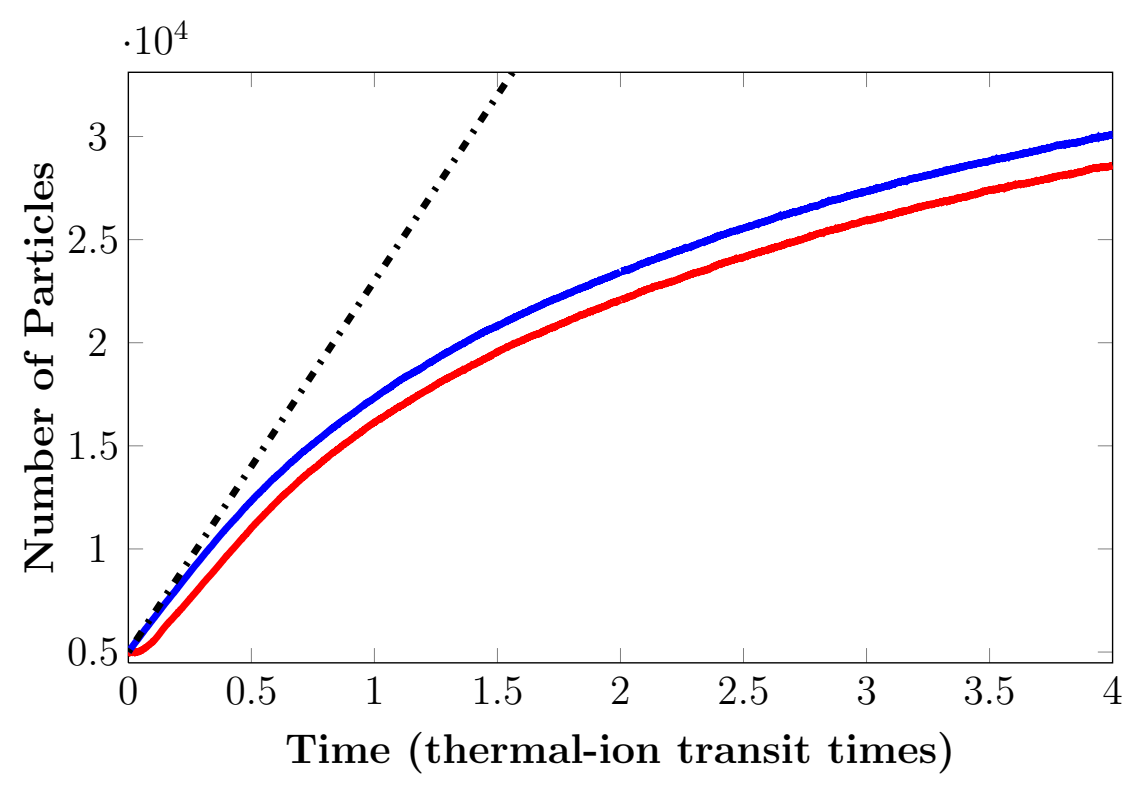

(b)

Figure 5: In 5a, we see the scalar potential profile at $t=3.6$ thermal-ion transit times. In 5b, we see the simulation electron and ion count, the red and blue curves respectively, along with the injection rate, the black dashed line. 


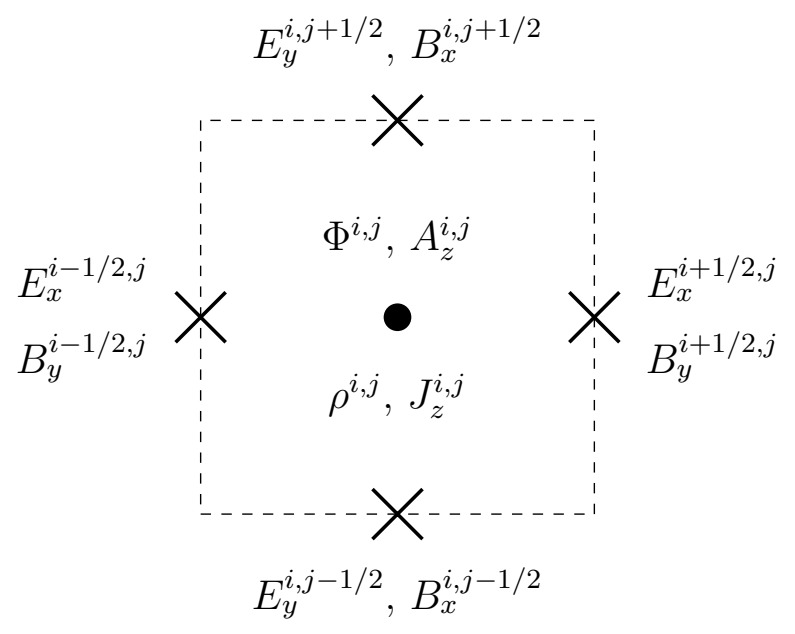

Figure 6: The staggered grid used for the Bennett pinch problem. 


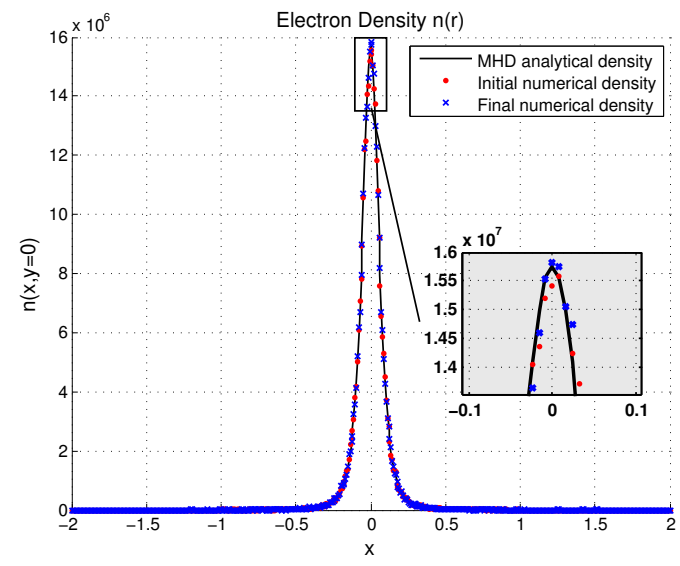

(a)

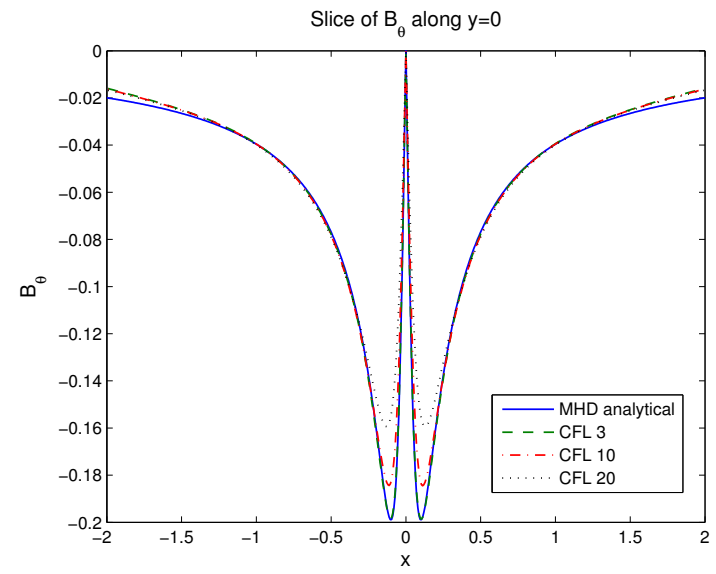

(c)

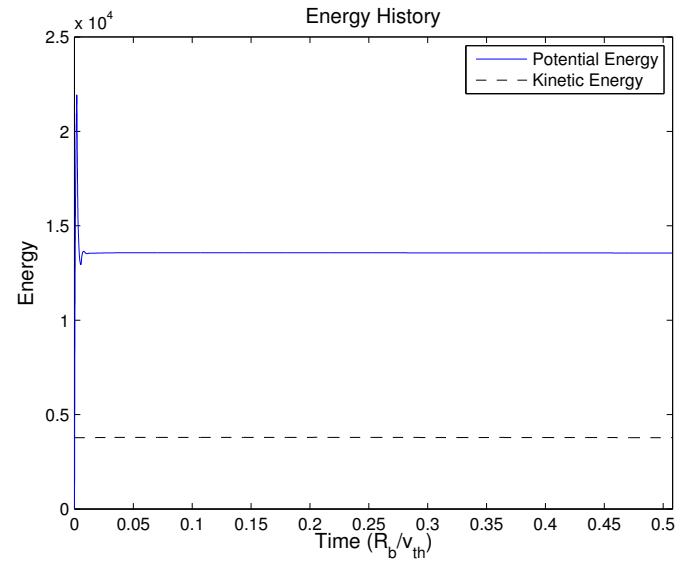

(b)

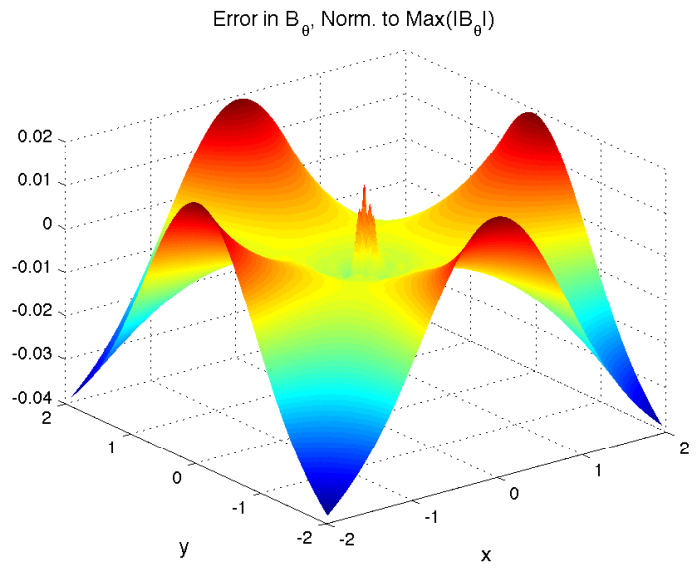

(d)

Figure 7: The figure shows 7a electron density, 7b and potential energies, 7c magnetic field at various CFL numbers, and $7 \mathrm{~d}$ the relative error in the azimuthal magnetic field $B_{\theta}$ (normalized to the maximum value of the magnetic field). Results are with a CFL number of 3, except as noted in 7 c. Position units are in terms of effective beam radius $R_{b}$. 


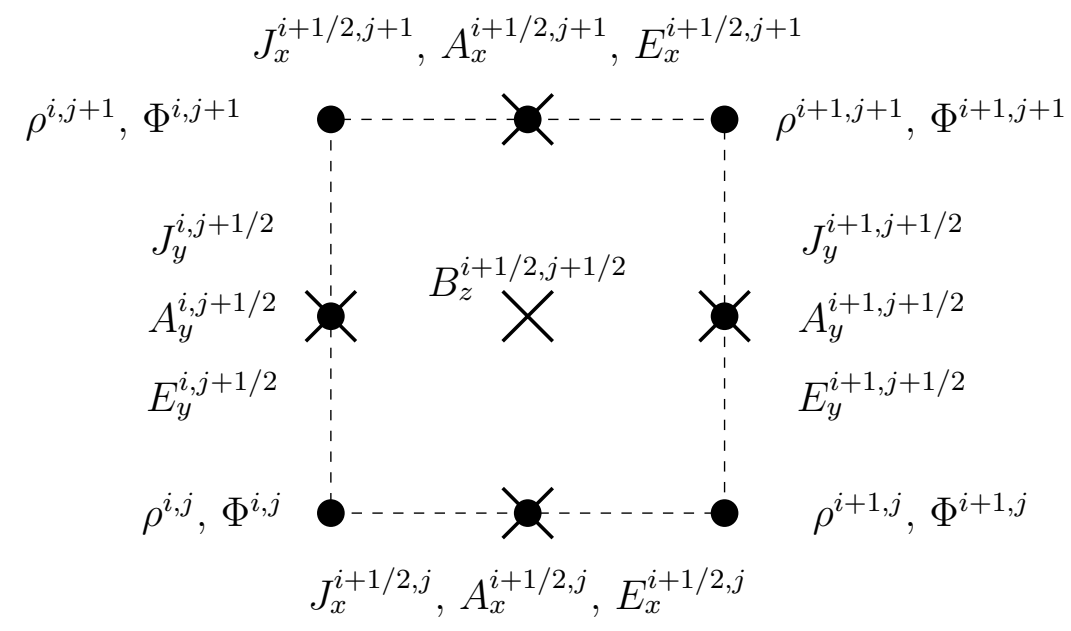

Figure 8: The staggered grid used for the Mardahl beam problem. 


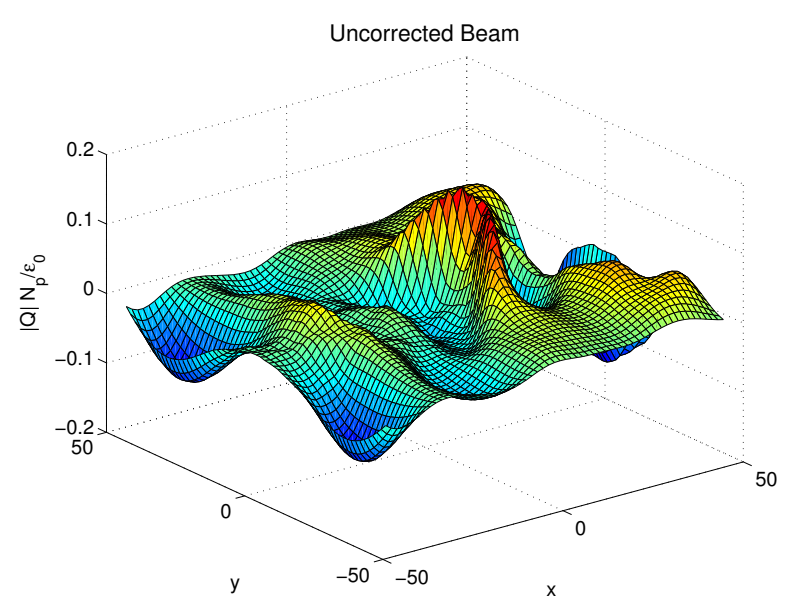

(a)

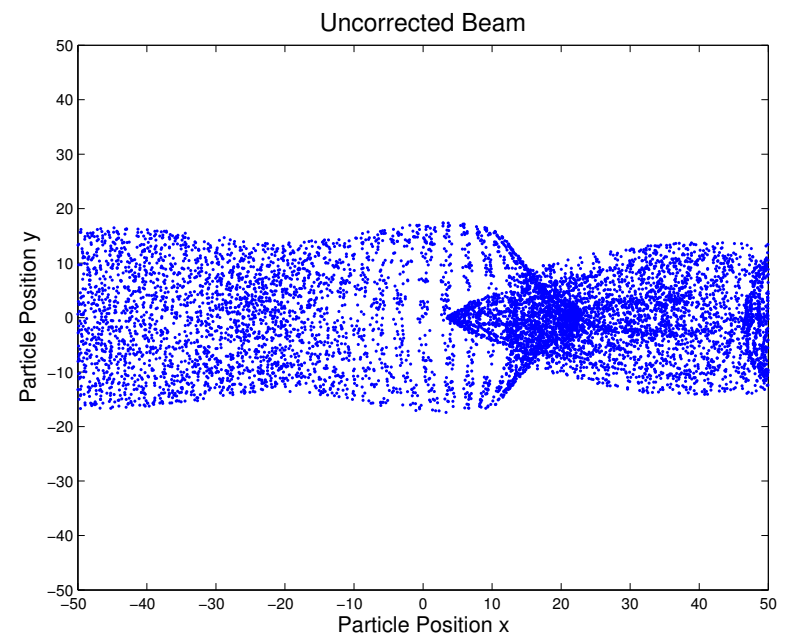

(c)

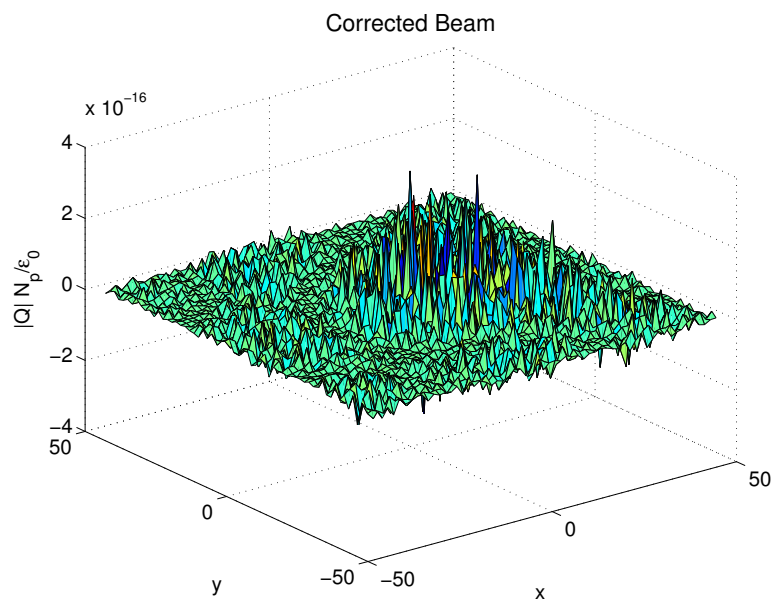

(b)

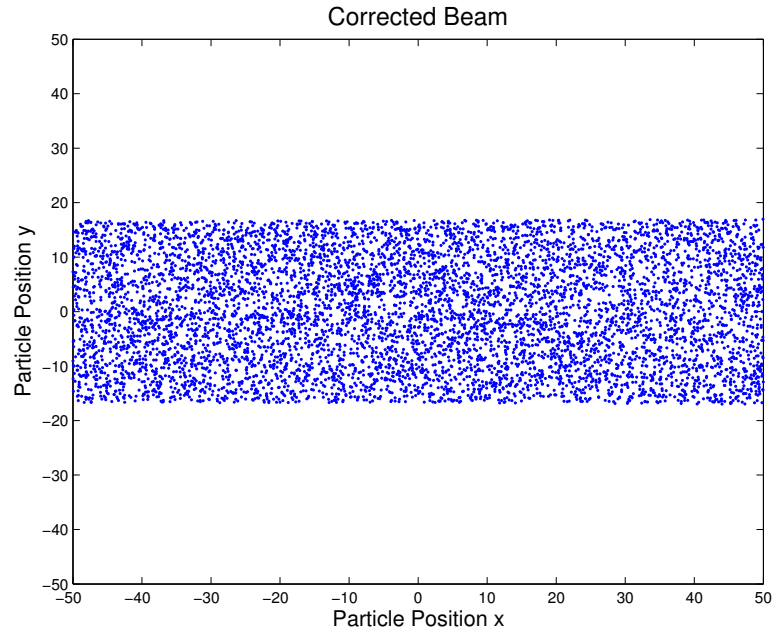

(d)

Figure 9: The figure shows the divergence error in the electric fields and the final beam distribution calculated from a wave equation potential 9a, 9c and in the Poisson equation potential 9b, 9d. 


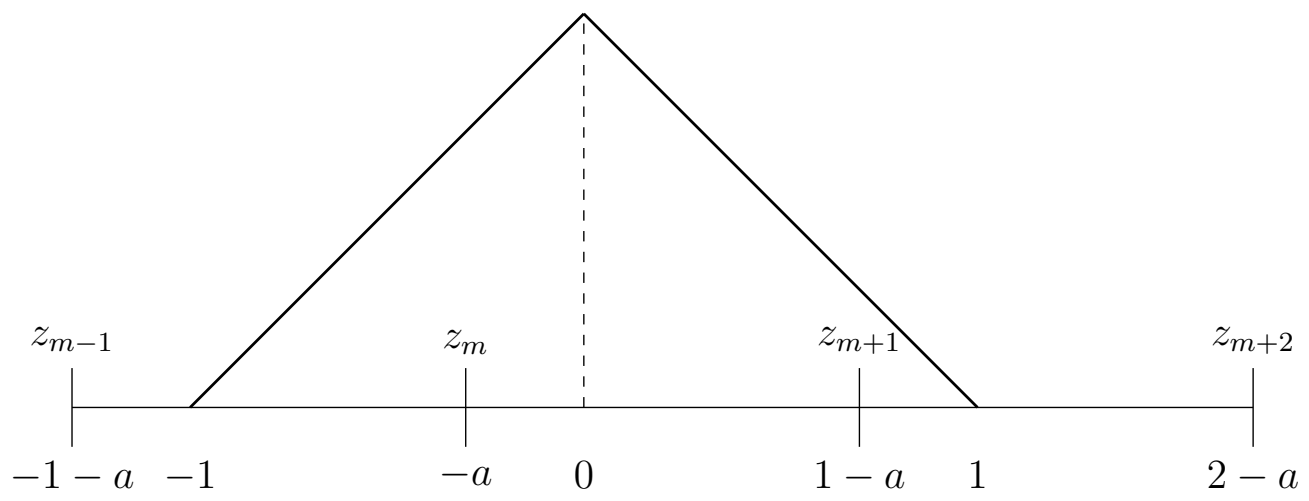

Figure C.10: $S(z)=1-|z|,|z|<1, S(z)=0,|z| \geq 1$

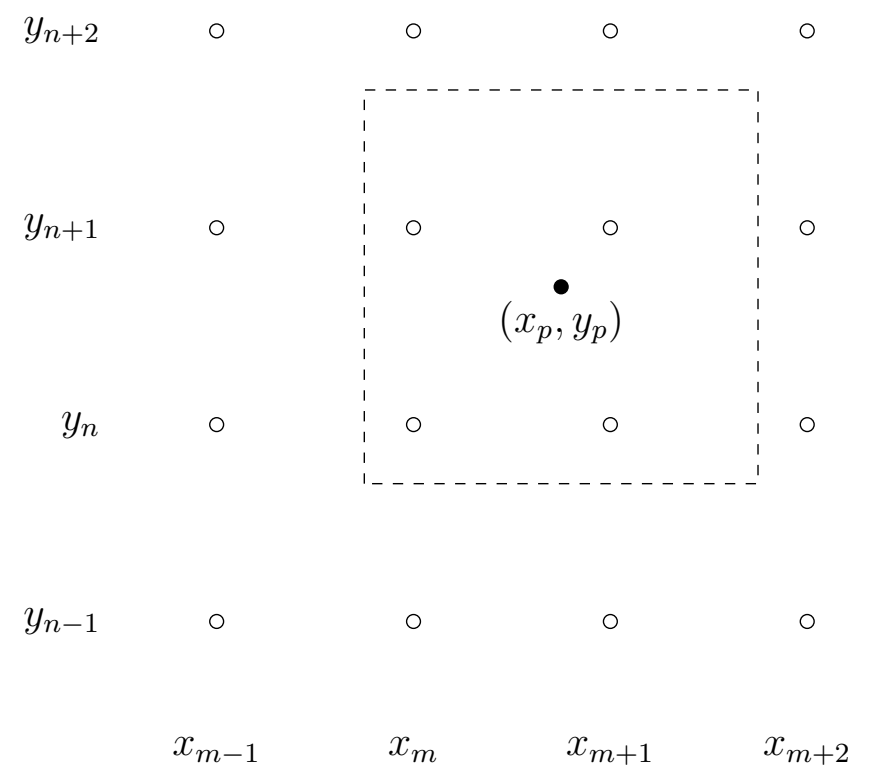

Figure C.11: The support of a linear particle shape $S_{p}(x, y)$ in $2 \mathrm{D}$. 